\title{
Macrophage depletion blocks congenital SARM1-dependent neuropathy
}

Caitlin B. Dingwall ${ }^{1,6}$, Amy Strickland ${ }^{1}$, Sabrina W. Yum², Aldrin K. Yim¹, Jian Zhu', Peter 5 L. Wang ${ }^{1,3}$, Yurie Yamada ${ }^{1}$, Robert E. Schmidt ${ }^{3}$, Yo Sasaki ${ }^{1}$, A. Joseph Bloom ${ }^{1,5}$, Aaron 6 DiAntonio ${ }^{4,5^{*}}$, Jeffrey Milbrandt ${ }^{1,5^{*}}$

${ }^{1}$ Department of Genetics, Washington University School of Medicine, St. Louis, United States.

$10 \quad{ }^{2}$ Children's Hospital of Philadelphia, Philadelphia, United States.

$11{ }^{3}$ Department of Pathology and Immunology, Washington University School of Medicine, 12 St. Louis, United States.

13 'Department of Developmental Biology, Washington University School of Medicine, St. 14 Louis, United States.

$15{ }^{5}$ Needleman Center for Neurometabolism and Axonal Therapeutics, St. Louis, United 16 States.

${ }^{6}$ MD-PhD Program, Washington University in St. Louis, St. Louis, United States.

*Correspondence to jmilbrandt@wustl.edu and diantonio@wustl.edu 


\section{ABSTRACT}

Axon loss contributes to many common neurodegenerative disorders. In healthy axons, the axon survival factor NMNAT2 inhibits SARM1, the central executioner of programmed axon degeneration. We identified two rare NMNAT2 missense variants in two brothers afflicted with a progressive neuropathy syndrome. The polymorphisms result in amino acid substitutions, V98M and R232Q, which reduce NMNAT2 NAD+-synthetase activity. We generated a mouse model of the human syndrome and found that Nmnat2 ${ }^{\mathrm{V} 98 \mathrm{M}} / \mathrm{Nmn}$ at $2^{\mathrm{R} 232 \mathrm{Q}}$ compound-heterozygous CRISPR mice survive to adulthood but develop progressive motor dysfunction, peripheral axon loss, and macrophage infiltration. These disease phenotypes are all SARM1-dependent. Remarkably, macrophage depletion therapy blocks and reverses neuropathic phenotypes in Nmnat2 ${ }^{\mathrm{V} 98 \mathrm{M} / \mathrm{R} 232 \mathrm{Q}}$ mice, identifying a SARM1-dependent neuroimmune mechanism as a key driver of disease pathogenesis. These findings demonstrate that SARM1 induces an inflammatory neuropathy and highlight the potential of immune therapy to treat this rare syndrome and other neurodegenerative conditions associated with NMNAT2 loss and SARM1 activation.

Key words: SARM1; axon; neuroinflammation; macrophages; NAD+; SARMopathy 


\section{INTRODUCTION}

Axon loss is one of the earliest pathological hallmarks and likely the initiating event in many common neurodegenerative disorders ${ }^{1-3}$. Programmed axon degeneration is an active, genetically encoded subcellular self-destruction pathway executed by the prodegenerative NADase enzyme SARM1 (sterile alpha and Toll/interleukin-1 receptor motifcontaining $)^{4,5}$. In a healthy axon, SARM1 activity is restrained by the NAD ${ }^{+}$biosynthetic enzyme NMNAT2, which converts NMN and ATP into NAD ${ }^{+6}$. NMNAT2 is a highly labile protein produced in the soma and trafficked into the axon ${ }^{7}$. Nerve injury blocks axonal transport and leads to rapid depletion of axonal NMNAT2 ${ }^{8}$, causing NMN buildup and $\mathrm{NAD}^{+}$loss. Recent breakthroughs led to the discovery that SARM1 is activated by an increase in the NMN to $\mathrm{NAD}^{+}$ratio ${ }^{9}$. NMN and NAD can both bind at an allosteric site in the enzyme's N-terminus to differentially regulate its conformation, and hence, the activation state of SARM1 ${ }^{9-11}$. The ratio of NMN/NAD ${ }^{+}$rises after NMNAT2 loss, favoring SARM1 activation and subsequent axon degeneration ${ }^{9}$. Genetic deletion of NMNAT2 alone is perinatal lethal; however, when combined with SARM1 deletion, mice are viable and resistant to injury-induced axon degeneration, suggesting that an essential role of NMNAT2 is to inhibit SARM1 ${ }^{12}$.

SARM1 is the central executioner of a cell-autonomous axon degeneration program. Upon activation, SARM1, an NAD hydrolase, depletes axonal $\mathrm{NAD}^{+}$levels, culminating in metabolic crisis and axon fragmentation. Acute injury is the best-understood trigger of pathological axon degeneration, inducing distal axon loss ${ }^{5,13-18}$. Loss of SARM1 is protective in models of chemotherapy-induced peripheral neuropathy (CIPN) ${ }^{14,16}$ and traumatic brain injury $(\mathrm{TBI})^{15}$. However, programmed axon degeneration is also common in chronic neurodegenerative disease models that do not include acute axonal injury ${ }^{14,16,19-25}$, suggesting a role for subacute SARM1 activation in the pathogenesis of a wide range of chronic neurodegenerative conditions.

Evidence for the involvement of the NMNAT2/SARM1 axon degeneration pathway in chronic disease has emerged from studying rare human patient mutations. Indeed, in a model of Leber congenital amaurosis type 9 (LCA9), SARM1 depletion rescues photoreceptor cell death caused by loss of the nuclear NMNAT isoform, NMNAT126. Furthermore, NMNAT2 mutations were identified in a still born fetus with fetal akinesia deformation sequence and two sisters suffering from a mild polyneuropathy ${ }^{27,28}$. The first direct evidence of SARM1 mutations in human disease emerged with the discovery of rare hypermorphic SARM1 alleles in a subset of ALS patients ${ }^{29,30}$. However, until the present study, the field lacked a mechanistically defined model of a SARM1-dependent, chronic axonopathy (termed "SARMopathy").

Here we examine two brothers that presented in early childhood with recurring GuillainBarré-like episodes requiring mechanical ventilation combined with severe, progressive peripheral neurodegeneration. Whole exome sequencing revealed they are both compound heterozygotes for two rare missense variants in the NMNAT2 gene, each inherited from one of their parents. We created a mouse that harbors both mutant NMNAT2 alleles and found that this model recapitulates key features of the human syndrome. NMNAT2 is the endogenous inhibitor of SARM1 in axons; thus, defects in NMNAT2 can trigger aberrant activation of SARM1. Indeed, we find that these disease phenotypes are all SARM1-dependent. While SARM1 is best understood as the executioner of a cell-autonomous axo-destructive program, here we make the surprising 
discovery that SARM1 induces axon degeneration via induction of non-cell-autonomous macrophage activation.

Macrophages play complex roles as both pro-degenerative and pro-restorative immune cells. Indeed, neuroinflammation has been referred to as a "double-edged sword" as it can have both beneficial and deleterious effects on the nervous system ${ }^{32}$. On one hand, in the peripheral nervous system, macrophages play a necessary role in facilitating axon regeneration through the clearance of myelin and axonal debris after nerve injury ${ }^{33-35}$. Indeed, macrophage depletion hampers axon regeneration after peripheral nerve injury ${ }^{36-}$ 39. However, macrophages and their CNS counterparts, microglia, are also drivers of disease in several common neurodegenerative diseases, including multiple sclerosis $(\mathrm{MS})^{40}$, chemotherapy-induced neuropathy (CIPN) ${ }^{41}$, and Alzheimer's Disease (AD $)^{42}$. In these disease models, depletion of macrophages and microglia can mitigate disease phenotypes, suggesting a conserved pro-degenerative role for phagocytes in human neurological disease.

Collectively, our data establish NMNAT2 variants as the genetic basis of a human neuropathy and demonstrate an unexpected role for SARM1 as a driver of neuroinflammation in the peripheral nervous system. In this model, we find that macrophage depletion early in the disease course can block the development of neuropathy, and remarkably, treatment after symptom onset can reverse neuropathy phenotypes. Our study provides the first mouse model of a chronic, injury-independent SARM1-dependent axonopathy ("SARMopathy") with which to test axon degenerationblocking treatments. This will be of substantial benefit and high clinical relevance as the field uncovers an ever-growing list of chronic neurodegenerative diseases that involve SARM1 activation. Importantly, our work uncovers a SARM1-dependent non-cellautonomous mechanism of axon loss and highlights macrophage depletion as a potent axo-protective therapeutic strategy.

\section{RESULTS}

\section{Rare missense variants in NMNAT2 cause hereditary neuropathy}

Patients 1 and 2 are brothers from non-consanguineous, healthy parents of African American ancestry. No further members of the extended family are known to be affected. Patient 1 was born following an uneventful pregnancy. Development was normal and the patient acquired the ability to walk before the onset of illness. At age 13 months, he experienced an acute episode of hypotonia, weakness, and respiratory failure requiring hospitalization and mechanical ventilation. Electrophysiology testing (nerve conduction studies and electromyography) at the time of symptom onset showed features of multifocal, sensory and motor neuropathy thought to be consistent with Guillain-Barre Syndrome. After treatment with intravenous immune globulin (IVIG) and steroids, he regained some motor function and was taken off ventilatory support but exhibited residual weakness.

In subsequent years he developed a unique sensorimotor syndrome comprised of both chronic and episodic features. Episodic attacks are frequently contemporaneous with infection and include severe neuropathic pain, worsening erythromelalgia, flaccid quadriparesis, and respiratory failure requiring mechanical ventilation. During these episodes, electrophysiological testing shows a complete absence of sensory and motor responses. In between episodes, the patient experiences a chronic, progressive motor- 
predominant peripheral neuropathy. Currently 25-years-old, Patient 1 is cognitively normal and attends college. Electrophysiology testing indicates a predominantly motor axonal neuropathy. He is wheelchair-dependent, exhibits progressive scoliosis, poor weight gain, and has severe combined proximal and distal muscle atrophy with predominantly distal muscle weakness (Fig. 1a). Muscle ultrasound revealed fatty, fibrotic tissue replacement of muscle, consistent with chronic neuropathy ${ }^{43}$. The patient also experiences recurring neuropathic pain, erythromelalgia, bilateral optic atrophy, and tongue fasciculation. Cranial and spinal MRI are normal except for mildly prominent extraaxial spaces. At age 15, a head CT showed mild diffuse parenchymal atrophy or pseudoatrophy. Also at 15, the patient had a normal echocardiogram and EKG testing indicated a possible enlarged atrium. Pulmonary function was tested, and spirometry demonstrated normal lung function.

Patient 2 was born 3 years after Patient 1 following an uneventful pregnancy. Patient 2 met early developmental milestones but experienced symptoms similar to his sibling prior to learning to walk. His first episode of severe weakness requiring mechanical ventilation occurred at 11 months. Patient 2's clinical course has been virtually identical to his brother's with very similar symptoms and degree of impairment.

Whole-exome sequencing was performed on the brothers and their parents to identify candidate variants that may cause the patients' disease. Both affected patients share rare, compound heterozygous variants [c. 695G>A (p.Arg232GIn) and c.292G>A (p.Val98Met)] in NMNAT2. Each parent was found to be heterozygous for one of the two variants identified in the patients (Fig. 1b). R232Q was previously identified as a loss-offunction variant associated with Fetal Akinesia Deformation Sequence and occurs in a region of NMNAT2 involved in substrate binding ${ }^{28}$. V98M is a novel NMNAT2 variant of unknown significance. Both variants occur at residues that are conserved in all three human NMNAT isoforms (Fig. 1c-d).

\section{V98M reduces NMNAT2 NAD ${ }^{+}$synthetase activity but not protein stability}

We sought to elucidate the functional consequences of the NMNAT2 variant alleles. To investigate whether NMNAT2 ${ }^{\mathrm{V} 98 \mathrm{M}}$ altered protein stability, we used immunoblotting to compare its relative half-life to NMNAT2 ${ }^{\mathrm{R} 232 \mathrm{Q}}$ and NMNAT2 in transfected HEK cells. In line with prior studies of NMNAT2 half-life ${ }^{8}$, protein synthesis blockade leads to a rapid drop in NMNAT2 protein levels. Turnover rates of NMNAT2 ${ }^{\mathrm{V} 98 \mathrm{M}}$ and NMNAT2 ${ }^{\mathrm{R} 232 \mathrm{Q}}$ are not significantly different from that of control NMNAT2 (Fig. 1e).

NMNAT2 is an NAD ${ }^{+}$synthesizing enzyme, and this activity is required for its function as an axon survival factor. To investigate whether NMNAT2 ${ }^{\mathrm{V} 9 \mathrm{M}}$ has impaired enzymatic activity, we purified recombinant Strep-tagged NMNAT2 proteins using affinity chromatography ${ }^{44}$. NMNAT2 ${ }^{\mathrm{V} 98 \mathrm{M}}$ has $14.6 \%$ of the $\mathrm{NAD}^{+}$synthesis activity of wild-type NMNAT2 at $37^{\circ} \mathrm{C}$, whereas NMNAT2 ${ }^{\mathrm{R} 232 \mathrm{Q}}$ is $4.4 \%$ as active as the wild-type enzyme, in agreement with previous findings ${ }^{28}$ (Fig. 1f). Collectively, these data demonstrate that these NMNAT2 variants disrupt enzymatic function, which may underly their pathogenicity.

\section{Nmnat2 ${ }^{V 98 M / R 232 Q}$ mice develop progressive motor neuropathy}

To study the pathological effects of the V98M and R232Q NMNAT2 mutations found in these patients, we used CRISPR-induced mutagenesis to create mice with mutations Nmnat2 ${ }^{V 98 M}$ or Nmnat2 ${ }^{R 232 Q}$ (see methods). Mice heterozygous for these individual 
206 mutations were mated to generate mice with compound heterozygous mutations Nmnat2 $2^{\mathrm{V} 98 \mathrm{M}}$ and $\mathrm{Nmnat} 2^{\mathrm{R} 232 \mathrm{Q}}$ (henceforth referred to as $N$ mnat2 ${ }^{\mathrm{V} 98 \mathrm{M} / \mathrm{R} 232 \mathrm{Q}}$ mice). These mutant mice were viable with no evidence of embryonic or perinatal lethality. As patients with compound heterozygous variants in NMNAT2 exhibit a chronic, motor-predominant peripheral neuropathy, we searched for similar phenotypes in the $N m n a t 2^{V 98 M / R 232 Q}$ mice. Starting at two months, we assayed muscle strength using an inverted screen test and found that the mice exhibit age-dependent, progressive muscle weakness (Fig. 2a). The human disorder involves predominantly distal muscle atrophy; therefore, we assayed hindlimb grip strength in the mutant mice. We observed a decline in distal muscle strength (Fig. 2b). Gait defects manifest in Nmnat2 ${ }^{V 98 M / R 232 Q}$ mice as young as six months old, concomitant with progressive lower limb muscular atrophy. The majority of mice display severe hindlimb wasting and difficulty walking by 9-12 months of age (Supp. Video 1). Notably, while the human patients have episodic neuropathic pain, we did not elicit a nociceptive defect in tail flick testing of $N$ mnat $2^{V 98 M / R 232 Q}$ mice (Fig. 2c).

\section{Nmnat2 ${ }^{\text {V98M/R232Q }}$ mice have electrophysiologic features consistent with a motor neuropathy}

The decreased muscle strength observed in Nmnat2 ${ }^{V 98 M / R 232 Q}$ mice suggests motor neuron dysfunction. We measured motor fiber function using compound muscle action potential (CMAP) amplitudes and found significant deficits in Nmnat2 ${ }^{V 98 M / R 232 Q}$ mice. The abnormalities worsen with age, suggesting that motor axon numbers progressively diminish in parallel with decreasing overall strength (Fig 2d-e). Next, we tested motor nerve conduction velocity (NCV). A decrease in NCV early in disease without altered CMAP amplitudes is indicative of demyelination, whereas a progressive drop in NCV concomitant with low CMAP amplitudes indicates large-diameter axon loss. The NCV in young Nmnat $2^{V 98 M / R 232 Q}$ mice is normal, indicating that the disease is primarily an axonal neuropathy; however, NCV does decrease with age, likely due to the eventual loss of large diameter axons (Fig. 2f). Electrophysiologic sensory testing demonstrated that large, myelinated sensory axons are not affected in Nmnat ${ }^{V 98 M / R 232 Q}$ mice (Fig $\mathbf{2 g}-\mathbf{h}$ ). Pain is transmitted by small and thinly myelinated fibers; thus nerve conduction studies are typically unaffected ${ }^{45}$. Rather, intraepidermal nerve fiber density (IENFD) analysis is a more sensitive measure of small fiber loss. In agreement with normal nociceptive function, we found that IENFD is unaffected in Nmnat2V98M/R232Q mice (Fig. S1). Altogether, these data demonstrate that $N$ mnat $2^{\mathrm{V} 98 M / R 232 Q}$ mice have a motor axonal neuropathy, consistent with the chronic electrophysiological features of human patients with NMNAT2-associated neuropathy.

\section{Nmnat2 variants cause progressive axon loss and muscle wasting in mice}

To further characterize the disease process in $N$ mnat $2^{\text {V98M/R232Q }}$ mice, we used light microscopic analysis to examine the pathology of select peripheral nerves including the sciatic (a mixed nerve), femoral (primarily motor), and sural (primarily sensory) in 2month, 6-month, and 9-12-month-old mice. The sciatic and femoral nerves exhibit severe, progressive axon loss. In contrast, we did not observe progressive axon loss in the sural nerve; however, total axon area was modestly different from wild-type control mice at 912 months of age (Fig. 3a-c). Myelin thickness is not affected in any of the nerves we examined (Fig. S2).

We next performed electron microscopic analysis on 2-month and 12-month-old Nmnat $2^{V 98 M / R 232 Q}$ sciatic nerves. The appearance of the sciatic nerve at two months is 255 normal and shows a dense population of large and small myelinated axons with little 
256 intervening extracellular space (Fig. 3d). Schwann cells and macrophages containing axonal and myelin debris are found in the endoneurial space (Fig. 3e, arrow). The sciatic nerve at 12 months shows patches of marked axon loss with increased collagen and wispy processes of Schwann cells (Fig. 3f, arrow). Following axon degeneration, perineurial cells take up lipid droplets from myelin breakdown ${ }^{46}$; indeed, we find large perineurial droplets of neutral fat in the 12-month sciatic nerve (Fig. 3g, arrow). To confirm that the observed peripheral defects are not due to motor neuron cell death, we immunostained spinal cords of 12-month-old wild-type and Nmnat $2^{V 98 M / R 232 Q}$ mice for the motor neuron marker choline acetyltransferase (ChAT) (Fig. 3h). Motor neuron cell numbers in the ventral horn are equivalent between genotypes, and thus, axon loss in Nmnat2 ${ }^{V 98 M / R 232 Q}$ nerves is likely not due to motor neuron cell death. Taken together, these pathological features demonstrate a progressive peripheral axonal neuropathy.

We next examined the neuromuscular junctions in the hindpaw lumbrical muscles of Nmnat $2^{V 98 M / R 232 Q}$ mice. We find the NMJ endplate size is slightly diminished in mutant mice even as early as 2 months of age (Fig. 3i, yellow arrows). The NMJ postsynaptic volume continues to progressively diminish over time, consistent with loss of presynaptic inputs (Fig. 3i-j). Apposition of the presynaptic nerve terminal and the postsynaptic endplate is a major determinant of NMJ functionality. Indeed, the ratio of overlap between presynaptic vesicles and the underlying acetylcholine receptor clusters (NMJ occupancy) is reduced in NMJs of Nmnat2 ${ }^{\mathrm{V} 98 M / R 232 \mathrm{Q}} 2$-month-old mice and decreases over time (Fig. $3 \mathbf{i}, \mathbf{k})$. In addition, endplate complexity is decreased in 12-month-old Nmnat2 ${ }^{\text {V98M/R232Q }}$ mice, whereas the prototypical pretzel-like endplate structure is still observed in 2-monthold Nmnat $2^{V 98 M / R 232 Q}$ mice (Fig. 3i, yellow arrows). Alterations in endplate size and complexity suggest repeated episodes of denervation and reinnervation. At steady state, the majority of $N m n a t 2^{V 98 M / R 232 Q}$ endplates appear partially innervated (Fig. 3i), however, almost all pre-terminal motor axons are abnormally thin and smooth (Fig. 3i, white arrows), a hallmark of sprouting axons ${ }^{47}$. Sprouting is frequently observed in NMJs of motor neuron disease models and is evidence of continual axonal degeneration and regeneration ${ }^{47-50}$. Taken together, these data indicate that decreased NMNAT2 activity causes progressive degeneration of terminal axons at the NMJ, consistent with a chronic motor neuropathy.

The patients with compound heterozygous variants in NMNAT2 have both proximal and distal weakness with predominantly distal muscle atrophy rendering them wheelchairbound. Loss of nerve terminals at the NMJ results in muscle fiber denervation and eventual muscle atrophy. Nmnat ${ }^{V 98 M / R 232 Q}$ mice exhibit a progressive reduction in hindlimb muscle mass (Fig. 3I) that correlates with decreased fiber cross-sectional area in the tibialis anterior muscle (Fig. $3 \mathrm{~m}$ ). Together, these results confirm that the decreased activity of the NMNAT2 mutants leads to axon loss and subsequent muscle denervation and atrophy. Importantly, the Nmnat $2^{V 98 M / R 232 Q}$ mouse model recapitulates chronic motor features of the human syndrome, providing strong evidence that the NMNAT2 variants are indeed pathogenic.

\section{Neuronal SARM1 is required for Nmnat2 ${ }^{V 98 M / R 232 Q}$ neuropathy}

SARM1 is a pro-degenerative enzyme activated by binding the NAD+ precursor NMN at its allosteric site ${ }^{9}$. NMNAT2 converts NMN to NAD ${ }^{+}$thereby preventing the buildup of NMN and its interaction with SARM1. In Nmnat2 KO mice, the increase in NMN leads to axon projection abnormalities and perinatal death; however, Nmnat2/Sarm1 double KO mice are viable and completely resistant to injury-induced programmed axon 
degeneration ${ }^{12}$. To determine whether SARM1 is activated in Nmnat2 ${ }^{V 98 M / R 232 Q}$ mice, we first monitored nerve levels of CADPR, a specific biomarker of SARM1 NAD ${ }^{+}$hydrolase activity $^{31}$. Metabolites were isolated from the sciatic nerve of two-month-old Nmnat2 ${ }^{V 98 M / R 232 Q}$ mice and analyzed by LC/MS-MS. We find that cADPR levels are elevated 8-fold compared to wild-type and this increase is fully SARM1-dependent (Fig. 4a), demonstrating that SARM1 is activated even at this early stage of the disease and suggesting that CADPR is likely an excellent biomarker for syndromes involving chronic SARM1 activation.

Next, we mated the Nmnat2 ${ }^{V 98 M / R 232 Q}$ mutant to Sarm1 KO mice to generate Nmnat2 ${ }^{V 98 M / R 232 Q}$; Sarm1 KO mice. In contrast with Nmnat2 ${ }^{V 98 M / R 232 Q}$ (Sarm1 WT) mice,

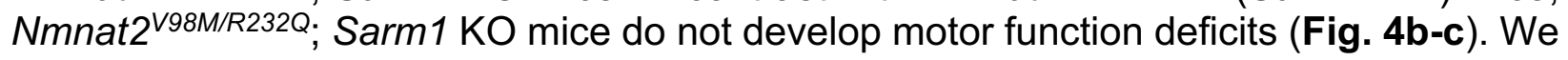
also performed morphological analysis of the sural, femoral, and sciatic nerves of Nmnat2 ${ }^{V 98 M / R 232 Q}$ and Nmnat2 ${ }^{V 98 M / R 232 Q}$; Sarm1 KO mice. As with the functional studies, loss of Sarm 1 prevents axon degeneration even in the oldest $N m n a t 2^{V 98 M / R 232 Q}$ mice (Fig. $3 \mathbf{a}, \mathbf{4 d - i )}$. These results confirm that the phenotypes associated with these pathogenic NMNAT2 variants are SARM1-dependent and do not arise secondary to neomorphic functions of the mutant NMNAT2 enzymes. These results suggest that Nmnat2 ${ }^{\text {V98M/R232Q }}$ mice will be useful for testing treatment strategies for progressive neurodegenerative disease involving chronic SARM1 activation.

The development of small molecule and gene therapy SARM1 inhibitors is underway ${ }^{22,51 ;}$ indeed, we previously showed that adeno-associated virus (AAV)-mediated, neuronspecific expression of a potent SARM1 dominant-negative (SARM1-DN) blocks pathologic axon degeneration in models of acute nerve injury ${ }^{22,52}$. With the discovery that the Nmnat2 ${ }^{V 98 M / R 232 Q}$ motor neuropathy is SARM1-dependent, we next tested whether this SARM1 gene therapy approach could similarly block disease in these mice. Onemonth-old Nmnat $2^{V 98 M / R 232 Q}$ mice received intrathecal injections of AAV virions $\left(6 \times 10^{11}\right)$ expressing SARM1-DN-EGFP or EGFP alone (control) under control of a synapsin promoter (Fig. 4j). We assayed inverted screen performance at 2 months and 6 months for both groups and determined therapeutic efficacy by comparing 6-month to 2-month performance for each mouse. Nmnat2 ${ }^{V 98 M / R 232 Q}$ mice injected with EGFP (control) display a $\sim 73 \%$ decline in strength by 6 months of age $(p<0.0001)$ (Fig. 4k). In contrast, Nmnat2 ${ }^{V 98 M / R 232 Q}$ mice injected with SARM1-DN exhibit a $39 \%$ decline (n.s., p>0.05) at 6 months of age (Fig. 4k). Importantly, SARM1-DN rescue of strength defects is dependent on extent of viral infection and transgene expression in the spinal cord. For example, higher expression correlates with higher endpoint performance, whereas lower expression correlates with lower endpoint performance (Fig. 4k, Fig. S3). These results demonstrate that neuron-specific high-level expression of SARM1-DN potently protects Nmnat2 ${ }^{V 98 M / R 232 Q}$ mice from developing motor deficits, demonstrating that neuronautonomous SARM1 activity is pathogenic in Nmnat2 ${ }^{V 98 M / R 232 Q}$ mice.

\section{Macrophages orchestrate Nmnat2 ${ }^{V 98 M / R 232 Q}$ neuropathy}

After acute nerve injury, macrophages infiltrate the lesion and phagocytose axonal and myelin debris, clearing the injury site and promoting axonal regeneration ${ }^{33-35}$. In models of chronic neurodegenerative disease, macrophages and their CNS counterpart, microglia, play complex immunomodulatory roles as both pro- and anti-inflammatory mediators in disease $41,42,53-59$. We immunostained central (spinal cord) and peripheral (sciatic nerve) nervous tissue with antibodies to the activated macrophage marker CD6860. There is a notable absence of $\mathrm{CD}^{6} 8^{+}$cells in central and peripheral tissues from 
wild-type mice (Fig. 5a, c). In contrast, in sciatic nerves of 2-month-old Nmnat2 ${ }^{\text {V98M/R232Q }}$ mice, $\mathrm{CD}^{+} 8^{+}$activated macrophages (Fig. 5e) are present, concomitant with a trend toward elevated $C D 64^{+} \mathrm{CD} 11 \mathrm{~b}^{+}$total nerve macrophages (Fig. 5f). Significantly fewer $\mathrm{CD} 68^{+}$macrophages are observed in Nmnat2 ${ }^{V 98 M / R 232 Q}$ mice lacking SARM1 at the same age (Fig. 5d), and the number of total nerve macrophages $\left(C D 64^{+} C D 11 b^{+}\right)$in these animals was similar to wild-type (Fig. 5f). CD68 $^{+}$macrophages are not detected in the spinal cords of Nmnat2 ${ }^{V 98 M / R 232 Q}$ mice (Fig. 5b), consistent with the predominantly peripheral nervous system defects in the patients. $\mathrm{NAD}^{+}$biosynthesis plays a role in programming macrophage immune responses ${ }^{61}$; thus, we examined whether intrinsic defects exist in Nmnat2 ${ }^{V 98 M / R 232 Q}$ macrophages due to impaired NMNAT2 activity. However, we found no differences in either basal or antigen-induced activation between wild-type and Nmnat2 ${ }^{V 98 M / R 232 Q}$-derived murine peritoneal macrophages (Fig. S3). This is consistent with findings in other disorders indicating that signals within the neural microenvironment shape macrophage phenotype activation ${ }^{62-66}$ as demonstrated by increased numbers of activated macrophages in $N$ mnat2 ${ }^{V 98 M / R 232 Q}$ sciatic nerves.

We performed bulk RNA Sequencing (RNA-Seq) on Nmnat2 ${ }^{\text {V98M/R232Q }}$ and wild-type (control) mouse sciatic nerves at early and late disease stages to capture dynamic changes in the glial and immune cell milieu (Fig. $\mathbf{5 g}$ ). Global transcriptomic analysis revealed similarities within $N$ mnat $2^{V 98 M / R 232 Q}$ nerves at both 2 months and 6 months, clustering more closely to each other than wild-type nerves. Closer inspection revealed sets of activated macrophage signatures ${ }^{62}$, including Cd68, Trem2, Apoe, Lrg1 and Ccl2, up-regulated in both 2-month and 6-month old Nmnat2 ${ }^{\text {V98M/R232Q }}$ mouse sciatic nerves (Fig. 5h), consistent with our observations of $\mathrm{CD}^{+} 8^{+}$nerve-associated macrophages in Nmnat2 ${ }^{\mathrm{V} 98 \mathrm{M} / \mathrm{R} 232 \mathrm{Q}}$ mice. Repair Schwann cell (SCs) markers ${ }^{67-69}$ such as Fgf5, Shh, Ngfr, and Olig1 are also upregulated, suggesting a dedifferentiation program of myelinating SCs. Gene ontology (GO) analysis showed significant enrichment of genes related to the immune response, inflammation, and phagocytosis signatures in both 2-month and 6month-old Nmnat2 ${ }^{V 98 M / R 232 Q}$ mice (Fig. 5i \& Supp. Tables 1-2). Altogether, these data demonstrate chronic activation of peripheral nervous system macrophages and an ongoing Schwann cell repair program in this mouse model.

Increased numbers of activated macrophages in the nerves of the Nmnat $2^{V 98 M / R 232 Q}$ mice raised the question of whether they are playing a beneficial or destructive role in the disorder. We used a macrophage depletion strategy using colony stimulating factor 1 receptor (CSF1R) blockade ${ }^{71,72}$ to evaluate the role of macrophages in Nmnat2 $2^{\text {V98M/R232Q }}$ mice. The mice were treated with CSF1R monoclonal antibody (or, isotype control antibody: IgG) every three weeks beginning at one month of age, and assessed using motor function tests at 2, 3, and 4 months of age (Fig. 6a). The efficacy of the macrophage depletion treatment was confirmed by immunostaining for CD68 in sciatic nerves (Fig. S4). Remarkably, macrophage depletion completely blocks the development of muscle strength defects for the duration of the experiment. In contrast, Nmnat2 ${ }^{V 98 M / R 232 Q}$ mice treated with isotype control antibody ( $\mathrm{lgG}$ ) continue to exhibit poor motor function (Fig. 6b). Morphological examination of the femoral (predominantly motor) nerve shows that macrophage depletion significantly rescues axon loss in the femoral nerve (Fig. 6c), consistent with its ability to prevent motor function deficits. Together, these data show that macrophages promote axon degeneration that leads to motor function deficits in this mouse neuropathy model.

Given our finding that macrophages contribute to axonal dysfunction early in the disease, 
we next tested whether macrophage depletion would be therapeutic after the initiation of symptoms. By four months of age, Nmnat2 ${ }^{V 98 M / R 232 Q}$ mice exhibit profound pathological and functional motor disease (Fig. 6b-c). Thus, we administered the CSF1R monoclonal antibody (or control IgG) to four-month-old Nmnat2 ${ }^{V 98 M / R 232 Q}$ mice to test whether macrophage depletion could halt or reverse disease progression (Fig. 6d). One month after antibody treatment, the previously symptomatic mice demonstrate a significant increase in inverted screen performance, demonstrating a profound recovery of muscle strength (Fig. 6e). Improvement in overall strength is accompanied by improved distal CMAP responses (Fig. 6f). Such a treatment response indicates that endogenous reparative processes occur after macrophage depletion to promote functional recovery. Indeed, in nerve and NMJ pathological studies, we detected ongoing axon degeneration and regeneration processes in $N$ mnat2 ${ }^{\mathrm{V} 98 \mathrm{M} / \mathrm{R} 232 \mathrm{Q}}$ mice at steady-state. Rescue of muscle strength persists until the mice are 7 months old, at which point muscle strength is maintained at pre-treatment levels. Endpoint examination of macrophage-depleted peripheral nerves reveals significant rescue of sciatic nerve axon loss and a trend toward rescue of femoral nerve axon loss (Fig. 6g). Altogether, these data support a model wherein macrophage depletion blocks axon degeneration, tipping the balance toward innate axon reparative processes and, thus, symptom reversal. Importantly, the surprising finding that macrophage depletion can reverse both behavioral and electrophysiologic defects underscores the potential of acute macrophage-targeted therapies in chronic neurologic diseases.

\section{DISCUSSION}

Genetic deletion of NMNAT2 is perinatal lethal; however, when combined with SARM1 deletion, mice are viable and resistant to injury-induced axon degeneration ${ }^{12}$. Recent advances in our understanding of the mechanisms underlying axon degeneration directly connected NMNAT2 loss to SARM1 activation through dynamic changes in the NMN-to$\mathrm{NAD}^{+}$ratio. These studies suggest that defects in NMNAT2 could predispose an individual to develop neurodegenerative disease; however, there is little direct evidence connecting mutations in the genes involved in axon degeneration to human neurological disease. Here, we examine two brothers with severe neuropathy associated with NMNAT2 mutations. This neuropathy initially presented with sensory and motor symptoms and progressed to be a predominantly motor neuropathy with severe muscle wasting. To study the molecular mechanism underlying this syndrome, we developed a mouse model harboring the $N$ mnat $2^{\mathrm{V} 98 M / R 232 Q}$ mutations. This mutant mouse recapitulates the cardinal motor features of the human syndrome. We find that NMNAT2 enzymatic deficiency leads to chronic SARM1 activation that, in turn, leads to non-cell-autonomous macrophage activation and axon loss. Our study has several important implications. First, the creation of our mouse model enables longitudinal examination of a SARM1dependent neuropathy, or SARMopathy, and provides a powerful platform for testing novel axo-protective therapeutics in disorders of chronic SARM1 activation. Second, we find that a major pro-degenerative role of SARM1 involves the activation of macrophages in parallel with its canonical cell-autonomous destructive functions. Finally, the identification of macrophages as key drivers of neuropathology suggests that macrophage depletion therapy could be efficacious in other diseases that involve SARM1-mediated axon degeneration. 
456 acutely damaged axons, there is growing evidence that this program is aberrantly activated in progressive neurodegenerative disease. Despite the abundance of acute injury models involving SARM1 activation, a chronic model that is more akin to human progressive neurodegenerative disorders is unavailable. Such a model is necessary for testing therapeutics under conditions of subacute SARM1 activation, which our work now shows involves previously unappreciated complex biological mechanisms. We find that SARM1 is activated at an early age in Nmnat2 ${ }^{V 98 M / R 232 Q}$ mice and that the disease is indeed fully SARM1-dependent. Moreover, this suggests that SARM1 activation and thus axon loss does not occur as an "all-or-nothing" event in chronic neurodegenerative disorders. Rather, this disorder is characterized by persistent SARM1 activity, suggesting that therapeutic intervention could be efficacious across a wide disease timeline in such disorders.

Patients with compound heterozygous variants $N M N A T 2^{V 98 M}$ and $N M N A T 2^{R 232 Q}$ develop a unique sensorimotor neuropathy involving both chronic and episodic symptomology. Chronic features of the disease are motor predominant, while episodic features involve prominent sensory and motor components. The patients typically develop acute episodes after infection, whereas the Nmnat2 ${ }^{V 98 M / R 232 Q}$ mouse model unsurprisingly shows no evidence of episodic disease features, likely due to housing in a pathogen-free environment. The mouse model does recapitulate cardinal features of the chronic symptomatology, including a motor axonal neuropathy, distal muscle wasting and weakness, and a progressive disease course. Unlike the patients, the Nmnat2 $2^{\text {V98M/R232Q }}$ mouse model has no evidence of a neuropathic pain phenotype, suggesting either species-dependent differences or that the episodic attacks seen in the patients but not in the mice contribute to the neuropathic symptoms. Interestingly, two sisters homozygous for a different variant in NMNAT2 (T94M) that largely retains enzymatic activity also display peripheral neuropathy, albeit significantly milder than the patients described in this study ${ }^{27}$.

From these studies in patients with the $N M N A T 2^{V 98 M / R 232 Q}$ mutations as well as these mutant mice, it appears that the peripheral nervous system is preferentially affected by NMNAT2 dysfunction, likely due to the longer length of peripheral axons. NMNAT2 has a very short half-life and is transported from the soma to the axon, thus distal regions are likely to be more sensitive to changes in NMNAT2 activity. Hence, the peripheral defects we observe are likely a result of axon length-dependent SARM1 activation. The NMNAT2/SARM1 axon degeneration pathway functions in both sensory and motor neurons; yet curiously, Nmnat2 ${ }^{V 98 M / R 232 Q}$ mice develop primarily a motor peripheral neuropathy. This is consistent with an Nmnat2 hypomorphic allele that also displays predominantly peripheral motor defects ${ }^{73}$. In contrast, in the Nmnat2 null mice, SARM1 drives loss of both sensory and motor nerves ${ }^{74}$, begging the question as to why motor neurons are preferentially affected in Nmnat $2^{V 98 M / R 232 Q}$ mice? Potentially, motor neurons are more vulnerable to chronic, low-level SARM1 activation, i.e. either due to a decreased tolerance for $\mathrm{NAD}^{+}$loss or increased susceptibility to macrophage-induced degeneration. In favor of this hypothesis, germline constitutive active SARM1 variants are enriched in human ALS patients ${ }^{29,30}$, suggesting that SARM1 primarily results in motor loss in vivo. Additional study of differential motor versus sensory axon susceptibility is required to answer these fundamental questions.

Studies of many common chronic neurodegenerative disorders have implicated the immune system as a key driver of disease, and activated macrophages are major 
contributors to tissue damage ${ }^{75}$. Similar to $\mathrm{CD} 8^{+}$microglia observed in Alzheimer's disease patients ${ }^{76}$ and LPS-induced central neuroinflammation ${ }^{77}$, CD68 ${ }^{+}$macrophages are abundant in the peripheral nervous system of $N$ mnat2 ${ }^{V 98 M / R 232 Q}$ mice, indicating they are activated. Importantly, macrophage activation begins early in the disease before significant axon loss has occurred, and in response to unknown signals within the neural microenvironment. We find that treating $N$ mnat2 ${ }^{V 98 M / R 232 Q}$ mice with AAV expressing a dominant-negative SARM1 molecule to prevent SARM1 activation specifically in neurons prevents motor dysfunction. Altogether, these data argue that macrophage activation occurs via an extrinsic SARM1-dependent signal rather than a macrophage-autonomous program in this model.

An unexpected finding of our study is that immunodepletion of macrophages both prevents and reverses Nmnat2-associated motor defects. Indeed, macrophage depletion in young mice blocks the development of a motor neuropathy in Nmnat2 ${ }^{V 98 M / R 232 Q}$ mice. Moreover, recovery of overall strength and improved nerve electrophysiology in older, macrophage-depleted $N$ mnat2 ${ }^{V 98 M / R 232 Q}$ mice demonstrate that axon dysfunction can be reversed in the presence of persistent SARM1 activation; however, this effect lessens as the disease progresses. While all recent mechanistic progress on SARM1 has defined it as the central driver of a cell-autonomous degenerative program via its $\mathrm{NAD}^{+}$hydrolase activity, our data support a new paradigm wherein chronic axonal SARM1 activation can also orchestrate non-cell-autonomous axon degeneration. Importantly, these findings indicate that macrophages are downstream effectors of SARM1 activation in vivo, placing SARM1 at the nexus between neuroinflammation and neurodegeneration.

So, how do macrophages induce axonal dysfunction in this syndrome? Evidence from studies of disease-associated microglia has established phagocytosis of live neurons, termed "phagoptosis", as contributing to CNS inflammation and neurodegeneration. In the present study, the absence of motor defects or axon loss after three months of macrophage depletion in young $N$ mnat $2^{V 98 M / R 232 Q}$ mice suggests that macrophages target axons that are either functional at baseline or have the ability to recover (i.e. stressedbut-viable) in the absence of macrophage attack. Pathologic evidence in the nerves and NMJs of Nmnat2 ${ }^{\mathrm{V} 98 \mathrm{M} / \mathrm{R} 232 \mathrm{Q}}$ mice point to a continual process of axon degeneration and regeneration, as has been observed in other chronic neurodegenerative diseases ${ }^{78,79}$. Moreover, reversal of motor deficits with macrophage depletion after disease onset suggests that there is effective and ongoing regeneration once axon degeneration is blocked.

Our data support a model wherein early axon loss and disease symptoms are due to macrophage activation and axon engulfment. We hypothesize that macrophages are key initiators of disease in this model of a SARM1-dependent motor neuropathy, akin to their previously described roles in other common neurodegenerative diseases including MS, $A D, P D$, and $A L S^{80}$. We predict that SARM1 activation eventually leads to neuronautonomous axon degeneration that is not prevented by immunomodulatory therapy. Indeed, this may explain the waning rescue offered by macrophage depletion in older Nmnat2 ${ }^{V 98 M / R 232 Q}$ mice. The findings herein demonstrate an acute benefit for use of currently available macrophage-targeted therapy. Given that Sarm1 knockout and SARM1 dominant negative gene therapy both confer robust protection in this model, use of future small molecule and gene therapy SARM1 inhibitors in combination with immune targeted therapies could be optimal for long-term therapy of chronic SARMopathies. 
556 While this study focuses on a very rare genetic cause of neuropathy, SARM1 has been 557 implicated in an expanding number of other, more common neurodegenerative diseases 558 including traumatic brain injury ${ }^{15,18,81}$, diabetic neuropathy ${ }^{16}$, chemotherapy-induced 559 neuropathy ${ }^{14,16,52}$, glaucoma ${ }^{25}$, and retinal degeneration ${ }^{26,82}$. Importantly, we and others 560 have identified rare hypermorphic human SARM1 alleles in patients with ALS ${ }^{29,30}$. Our 561 work suggests that in addition to SARM1 inhibition, these patients are also candidates for 562 macrophage targeted therapies. In summary, our study implies the existence of a 563 SARM1-dependent, non-cell-autonomous pathway for axonal dysfunction that is 564 amenable to targeted immunomodulatory therapy and presents a mechanistically defined 565 mouse model of a pure SARMopathy in which to test such treatment strategies. 


\section{MATERIALS AND METHODS}

\section{Whole-exome sequencing}

Whole exome sequencing and sequence analysis were performed by the Division of Genomic Diagnostics Genetic Counseling Core at Children's Hospital of Philadelphia. Genomic DNA was extracted from peripheral blood following standard DNA extraction protocols. Targeted exons were captured with the Agilent SureSelect XT Clinical Research Exome kit per manufacturer's protocol, sequenced on the Illumina HiSeq 2000 or 2500 platform with 100bp paired-end reads, and sequencing variants were identified using an in-house custom-built bioinformatics pipeline as described previously ${ }^{83}$. Mapping and analysis were based on the human genome build UCSC hg19 reference sequence. Single nucleotide variants, small deletions, and small insertions were detected. Suspected pathogenic variants were confirmed by Sanger sequencing.

\section{Constructs}

The Takara HD InFusion Cloning Kit was used to introduce R232Q and V98M mutations into the complete open reading frame of the canonical 307 amino acid human NMNAT2 reference allele fused to a FLAG tag followed by a 10 amino acid linker sequence and Cterminal 2 X Strep Tag cloned into lentivirus vector FCIV.

\section{HEK293T transfection}

HEK293T cells were cultured in DMEM with $2 \mathrm{mM}$ glutamine and $1 \%$ penicillin/streptomycin (both Gibco), and 10\% fetal bovine serum. Cells were plated in 12well format to reach $25-35 \%$ confluence before transfection with polyethyleneimine (PEI, $1 \mathrm{mg} / \mathrm{mL}, \mathrm{pH} 7.0$ ) using a ratio of $3: 1$ (mg of PEl vs. mg of plasmid DNA). $200 \mathrm{ng}$ StrepTag-NMNAT2-Flag expression vectors (reference allele, V98M, or R232Q) were transfected per well. $1 \mathrm{mg} / \mathrm{mL}$ Cycloheximide (Sigma-Aldrich) was used to block protein synthesis 24 hours after transfection. Cells from a single well at each time point after treatment were suspended in $100 \mathrm{~mL}$ of $100 \mathrm{mM}$ Tris-Cl, $\mathrm{pH} 8.0$, and $150 \mathrm{mM} \mathrm{NaCl}$ with Protease Inhibitor Cocktail (Pierce, Buffer 1), sonicated to fragment genomic DNA, then mixed with $30 \mathrm{~mL}$ of $4 \times$ NuPage LDS sample buffer (Invitrogen). After heating to $90^{\circ} \mathrm{C}$ for 5 minutes, equal amounts were used for Western blot.

\section{Western Blot}

Cell lysates were resolved using SDS polyacrylamide gel electrophoresis (PAGE) on 4$12 \%$ Bis-Tris Plus gel (Invitrogen), transferred to PVDF membrane (Invitrogen), and followed by immunoblotting for Flag (1:1000 Mouse Anti-Flag M2 monoclonal, Sigma F3165) and beta-tubulin (1:1000 Anti-beta-tubulin DSHB Clone E7), and visualization by standard chemiluminescence.

\section{NMNAT recombinant protein expression and purification}

Constructs described above, human reference allele of NMNAT2 and variants V98M or $\mathrm{R} 232 \mathrm{Q}$, were transfected to $150 \mathrm{~mm}$ diameter cell culture dish with $\sim 50 \%$ confluence of HEK293T cells. $15 \mathrm{mg}$ of plasmid was mixed with $45 \mathrm{mg}$ of PEI and transfected into the cells. Cells were harvested 48 hours after transfection and resuspended in Buffer 1 before lysis by sonication on ice. After centrifugation at $18,000 x g$ for 10 minutes 3 times to remove the cell debris, the supernatant was mixed with PureCube HiCap Streptactin MagBeads (Cube Biotech) for 1 hour. Proteins were eluted with Buffer 1 plus $5 \mathrm{mM}$ desthiobiotin and stored at $-80^{\circ} \mathrm{C}$. Their concentrations were determined by the Bio-Rad 
staining.

\section{NMNAT Activity Assay}

NMNAT activity was defined as $1 \mathrm{mmol} \mathrm{NAD}^{+}$generated per min (one unit, $U$ ) per mg of protein. Typically, the reaction was initiated by mixing purified NMNAT2 protein at 1-2 $\mathrm{mg} / \mathrm{mL}$ with $100 \mathrm{mM}$ ATP and $100 \mathrm{mM}$ NMN in $10 \mathrm{mM}$ HEPES, $\mathrm{pH} 7.4$, and $5 \mathrm{mM} \mathrm{MgCl} 2$ at $37^{\circ} \mathrm{C}$. At various time points, reactions were stopped by removing $100 \mu \mathrm{L}$ from the reaction and mixing it with $100 \mu \mathrm{L} 0.5 \mathrm{M}$ perchloric acid $(\mathrm{HClO} 4)$ on ice for 10 minutes. After centrifugation at $18,000 \mathrm{xg}$ for 10 minutes, the supernatant was mixed with $11 \mu \mathrm{L} 3$ M K2CO3 for neutralization. Samples were placed on ice for another 10 minutes and centrifuged. $45 \mu \mathrm{L}$ of supernatant containing extracted metabolites was mixed with $5 \mu \mathrm{L}$ $0.5 \mathrm{M}$ Potassium Phosphate buffer and quantified by HPLC (Nexera X2) on a Kinetex $\left(100 \times 3 \mathrm{~mm}, 2.6 \mu \mathrm{m}\right.$; Phenomenex) column. An NAD ${ }^{+}$standard (Sigma) was used to generate a standard curve for the quantification of $\mathrm{NAD}^{+}$in the extraction. NAD ${ }^{+}$ production at 10 minutes, which is within the steady-state, was used to calculate NMNAT activity.

\section{Enzymatic and half-life assay statistics} Data are presented as Mean \pm SEM. Fitting of data was performed using Excel (Microsoft) or Prism 9 (GraphPad). Curve fittings and specific tests used are described in the Figure legends. A $p$-value $<0.05$ was considered significant.

\section{Generation of Nmnat2 ${ }^{V 98 M / R 232 Q}$ compound heterozygous mice}

All animal experiments were performed under the direction of institutional animal study guidelines at the Washington University, St. Louis, MO. To generate Nmnat2 ${ }^{V 98 M / R 232 Q}$ mice via CRISPR/Cas9, guide RNAs were designed by the Washington University School of Medicine Genome Engineering \& iPSC Center, and individually created gRNAs were microinjected into B6CBAF2/J pronuclei along with Cas9 protein and donor DNA oligonucleotides engineered to introduce the desired mutations by homology-directed repair. Properly mutated transgenic mice with individual mutations were confirmed by sequencing. Founder mice were initially mated to C57BL/6J mice and heterozygotes for each allele were subsequently mated together to generate trans-heterozygotes.

\section{Nerve structural analysis}

Sciatic, sural, and femoral nerves were processed as previously described ${ }^{14,84}$. Briefly, nerves were fixed in $3 \%$ glutaraldehyde in $0.1 \mathrm{mlPBS}$ (Polysciences) overnight at $4{ }^{\circ} \mathrm{C}$, washed, then stained with $1 \%$ osmium tetroxide (Sigma Aldrich) overnight at $4^{\circ} \mathrm{C}$. Nerves were then washed, and dehydrated in a serial gradient of ethanol from 50 to $100 \%$. After dehydration, nerves were incubated in 50\% propylene oxide $/ 50 \%$ ethanol, then $100 \%$ propylene oxide. Subsequently, nerves were incubated in the Araldite resin solution/propylene oxide solutions overnight in the following ratios: 50:50, 70:30, 90:10, and embedded in 100\% Araldite resin solution (Araldite: DDSA: DMP30; 12:9:1; Electron Microscopy Sciences) and baked overnight at $60^{\circ} \mathrm{C}$. Sciatic nerves were embedded so that the most distal portion was sectioned. For the light microscope analysis of 400$600 \mathrm{~nm}$ semithin sections were cut using Leica EM UC7 Ultramicrotome and placed onto microscopy slides. Toluidine blue staining and quantification were performed as previously described ${ }^{84}$. All quantifications were performed by an individual blinded to genotype.

\section{Tissue immunohistochemistry}




\section{Nervous tissue}

Six micron-thick sections of sciatic nerves and twenty micron-thick sections of spinal cord were prepared on a cryostat (Leica CM1860), mounted onto slides, and processed as described ${ }^{14}$. For visualization of activated macrophages, the primary antibody used was 1:100 CD68 (rat, Bio-Rad), and the secondary antibody was anti-rat Cy3 (Jackson Immunoresearch) at a dilution of 1:500. For visualization and quantification of GFP fluorescence in spinal cords, directly conjugated rabbit anti-GFP Alexa 488 (Invitrogen) was used 1:200. For visualization of motor neuron cell bodies, the primary antibody used was 1:100 ChAT (rat, Millipore), and the secondary antibody was 1:250 anti-goat-Cy3 (Jackson Immunoresearch). Sections were cover-slipped with Vectashield with DAPI (Vector Laboratories) to allow visualization of nuclei. Sciatic nerves were imaged with the Leica DMI 4000B confocal microscope using a 40x immersion oil. Spinal cords were imaged at $10 x$ magnification using a scheme covering $100 \%$ of the total spinal cord area with image stitching for quantification. Z-stacks were acquired through the whole sample, max projected, then exported for quantification. ImageJ was used for quantification of integrated fluorescence density quantification from three independent sections per mouse. Motor neuron numbers were manually counted in the ventral horn using ImageJ (3-4 mice per genotype).

\section{Tibialis Anterior Muscle}

Ten micron-thick sections of the tibialis anterior muscle were processed as described ${ }^{14}$. The primary laminin antibody was used at 1:100 (Sigma \#L9393). The secondary antibody was 1:500 anti-rabbit Cy3 (Jackson Immunoresearch). Muscle fiber crosssectional area was calculated by averaging fiber cross-sectional area from $\geq 150$ muscle fibers per mouse from three independent 20X images. Three mice were used per genotype in each age cohort.

\section{Lumbricals}

The mice were transcardially perfused with $20 \mathrm{ml}$ of $4 \%$ paraformaldehyde (PFA) solution. Dissected lumbrical muscles were permeabilized with $2 \%$ TritonX-100 /1x PBS (PBST) for $30 \mathrm{~min}$ and then blocked with $4 \%$ bovine serum albumin (BSA) dissolved in $0.3 \%$ PBST $30 \mathrm{mins}$ at room temperature. Muscles were incubated with primary antibodies of SV2 (1:200, DSHB AB2315387) and $2 \mathrm{H3}$ (1:100, DSHB AB2314897) overnight at $4^{\circ} \mathrm{C}$. After incubation of primary antibody, muscles were incubated with FITC rabbit anti-mouse IgG1 (1:400, Invitrogen A21121) and Alexa fluoro-568 conjugated $\alpha$-bungarotoxin (1;500, biotium 00006) for $2 \mathrm{hrs}$ at room temperature. Muscles were washed with PBS for 5 min 3 times and then were mounted by mounting media (VECTASHIELD).

To analyze NMJ morphology, z-stack images were obtained. Maximal intensity projection images were analyzed to determine the volume of endplates using Imaris software. 1520 NMJs were analyzed per mouse. NMJ occupancy was calculated as a ratio of presynaptic axonal area (SV2/2H3) versus post-synaptic area (BTX). As the value approaches 1.0, it indicates motor endplates are fully occupied by axons.

\section{Intraepidermal nerve fiber density quantification}

Intraepidermal nerve fiber (IENF) staining and quantification were performed as previously described ${ }^{14,85}$. Briefly, footpad skin was dissected and then placed in freshly prepared Zamboni's fixative overnight at $4^{\circ} \mathrm{C}$. Samples were then thoroughly washed with PBS then incubated for $24 \mathrm{hr}$ in $30 \%$ sucrose in PBS. Samples were embedded in O.C.T. then sectioned at $50 \mu \mathrm{m}$ using a cryostat. Sections were stored $-20^{\circ} \mathrm{C}$ in a cryoprotectant 
comprised of $30 \%$ sucrose and $33 \%$ ethylene glycol in PBS until ready for immunohistochemistry. Free floating sections were washed with PBS, blocked with $5 \%$ normal goat serum in PBST, then incubated in PGP9.5 (AB1761, Millipore) diluted 1:1000 in blocking buffer overnight at $4^{\circ} \mathrm{C}$. Free floating sections were washed with PBST, incubated with species appropriate secondary antibody (anti-rabbit-Cy3) 1:500 for $2 \mathrm{hr}$ at room temperature, washed with PBST, and then mounted in Vectashield with DAPI.

The footpads were identified and imaged on a Leica DMI 4000B confocal microscope using a $10 \times$ objective. Z-stacks were acquired through the whole sample, max projected, then exported for quantification. IENF density was quantified as the number of PGP9.5 axons that crossed the basement membrane normalized to the length of the basement membrane. IENF densities were averaged in three separate sections for each animal. Imaging and analysis were performed by an individual blinded to genotype.

\section{Tissue metabolite measurements}

732 On the day of metabolite extraction, sciatic nerve tissues were homogenized in $160 \mu \mathrm{L}$ of cold $50 \% \mathrm{MeOH}$ solution in water using a homogenizer (Branson) and then centrifuged $\left(15,000 \mathrm{~g}, 4^{\circ} \mathrm{C}, 10 \mathrm{~min}\right)$. The clear supernatant was transferred to a new tube containing $50 \mu \mathrm{L}$ chloroform and vigorously shaken then centrifuged (15,000 g, $\left.4^{\circ} \mathrm{C}, 10 \mathrm{~min}\right)$. The chloroform extraction was repeated twice. The clear aqueous phase $(100 \mu \mathrm{L})$ was transferred to the new tube and then lyophilized and stored at $80^{\circ} \mathrm{C}$ until measurement. Lyophilized samples were reconstituted with $50 \mu \mathrm{L}$ of $5 \mathrm{mM}$ ammonium formate (Sigma) and centrifuged at $12,000 \times \mathrm{g}$ for $10 \mathrm{~min}$. Cleared supernatant was transferred to the sample tray. Nervous tissue metabolite measurements were acquired as previously described $^{84}$.

\section{AAV constructs and virus injections} AAV constructs were created as previously described ${ }^{22}$. Briefly, AAV8-hSYN-SARM1DN-EGFP was generated by the viral vector core of the Hope Center for Neurological Disorders at Washington University in St. Louis. Viral particles were purified by iodixanol gradient ultracentrifugation, and virus titers were measured by dot blot. Under light anesthesia with Avertin, $6 \times 10^{11}$ viral genomes were injected intrathecally at L6/S1. Viral expression was confirmed by detecting EGFP expression via immunohistochemical analysis of sectioned spinal cords.

\section{Nerve electrophysiology}

Compound muscle action potentials (CMAPs) were acquired as previously described ${ }^{86}$ using a Viking Quest electromyography device (Nicolet). Mice were anesthetized, then electrodes (stimulating: ankle or sciatic notch; recording: footpad) were put into place. Supramaximal stimulation was used for CMAPs. SNAPs were acquired as previously described ${ }^{14}$ using a Viking Quest electromyography device (Nicolet). Briefly, mice were anesthetized, then electrodes were inserted subcutaneously into the tail (recording electrode at the base, stimulating electrodes $30 \mathrm{~mm}$ distal from the negative recording electrode, and ground between the stimulating and recording electrodes). Supramaximal stimulation was used for SNAPs.

764 The tail flick assay was performed as previously described ${ }^{87}$. Briefly, mice were restrained 765 horizontally, and the tip of their tails was submerged in a $55^{\circ} \mathrm{C}$ water bath. All mice 
766

767

768

769

770

771

772

773

774

775

776

777

778

779

780

781

782

783

784

785

786

787

788

789

790

791

792

793

794

795

796

797

798

799

800

801

802

803

804

805

806

807

808

809

810

811

812

813

814

815

removed their tails from the noxious stimulus by flicking their tails out of the water. The latency to withdrawal from the water bath was measured using a stopwatch. Five trials were performed and averaged for each mouse.

\section{Inverted screen assay}

The inverted screen assay was performed as previously described with minor modifications ${ }^{88}$. Mice were placed on a wire mesh screen. The latency to fall for each mouse was recorded, and each mouse underwent three trials with five-minute rest periods. If a mouse did not fall off the mesh screen within 120 seconds, then that time was recorded, and the mouse was taken off the screen.

\section{Hindlimb grip strength}

Hindlimb grip strength was measured using a computerized grip strength meter. To measure hindlimb grip strength, mice were held upright at the base of the neck and the tail, and the mouse was held over a bar connected to a force transducer. Once the mouse gripped the bar with its hind paws, the mouse was pulled backward until the grip was released. The maximum force of each measurement was measured in grams and recorded by the grip strength meter. Each mouse underwent five trials. If a mouse could not establish a grip on the bar, then the value for that trial was recorded as zero.

\section{Flow cytometry}

Nerves were collected and kept on ice until dissociation. Cells were then minced and incubated with gentle shaking for $20 \mathrm{~min}$ in digestion media containing $0.3 \%$ collagenase IV, $0.04 \%$ hyaluronidase, and $0.04 \%$ Dnase in DMEM at $37^{\circ} \mathrm{C}$. Cells were then washed and filtered through $70 \mu \mathrm{m}$ cell strainers. Single-cell suspensions were stained at $4{ }^{\circ} \mathrm{C}$. Dead cells were excluded by propidium iodide $(\mathrm{PI})$. Antibodies to the following proteins were used: CD11b (clone M1/70), CD45 (clone 30-F11), and CD64 (clone X54-5/7.1). Cells were analyzed on an LSRII flow cytometer (Becton Dickinson) and analyzed with FlowJo software.

\section{RNA Sequencing and Analysis}

Samples were prepared according to library kit manufacturer's protocol, indexed, pooled, and sequenced on an Illumina NovaSeq 6000. Basecalls and demultiplexing were performed with Illumina's bcl2fastq software and a custom python demultiplexing program with a maximum of one mismatch in the indexing read. RNA-seq reads were then aligned to the Ensembl release 76 primary assembly with STAR version $2.5 .1 \mathrm{a}^{1}$. Gene counts were derived from the number of uniquely aligned unambiguous reads by Subread:featureCount version 1.4.6-p52. Isoform expression of known Ensembl transcripts were estimated with Salmon version $0.8 .2^{3}$. Sequencing performance was assessed for the total number of aligned reads, total number of uniquely aligned reads, and features detected. The ribosomal fraction, known junction saturation, and read distribution over known gene models were quantified with RSeQC version 2.6.24.

All gene counts were then imported into the R/Bioconductor package Edge ${ }^{5}$ and TMM normalization size factors were calculated to adjust for samples for differences in library size. Ribosomal genes and genes not expressed in the smallest group size minus one samples greater than one count-per-million were excluded from further analysis. The TMM size factors and the matrix of counts were then imported into the R/Bioconductor package Limma ${ }^{6}$. Weighted likelihoods based on the observed mean-variance relationship of every gene and sample were then calculated for all samples and the count 
816 matrix was transformed to moderated $\log 2$ counts-per-million with Limma's voomWithQualityWeights ${ }^{7}$. The performance of all genes was assessed with plots of the residual standard deviation of every gene to their average log-count with a robustly fitted trend line of the residuals. Differential expression analysis was then performed to analyze for differences between conditions and the results were filtered for only those genes with Benjamini-Hochberg false-discovery rate adjusted $p$-values less than or equal to 0.05 .

For each contrast extracted with Limma, global perturbations in known Gene Ontology (GO) terms, MSigDb, and KEGG pathways were detected using the R/Bioconductor package $\mathrm{GAGE}^{8}$ to test for changes in expression of the reported log 2 fold-changes reported by Limma in each term versus the background log 2 fold-changes of all genes found outside the respective term. The R/Bioconductor package heatmap3 ${ }^{9}$ was used to display heatmaps across groups of samples for each GO or MSigDb term with a Benjamini-Hochberg false-discovery rate adjusted p-value less than or equal to 0.05 . Perturbed KEGG pathways where the observed log 2 fold-changes of genes within the term were significantly perturbed in a single-direction versus background or in any direction compared to other genes within a given term with $p$-values less than or equal to 0.05 were rendered as annotated KEGG graphs with the R/Bioconductor package Pathview $^{10}$.

To find the most critical genes, the Limma voomWithQualityWeights transformed log 2 counts-per-million expression data was then analyzed via weighted gene correlation network analysis with the R/Bioconductor package WGCNA ${ }^{11}$. Briefly, all genes were correlated across each other by Pearson correlations and clustered by expression similarity into unsigned modules using a power threshold empirically determined from the data. An eigengene was then created for each de novo cluster and its expression profile was then correlated across all coefficients of the model matrix. Because these clusters of genes were created by expression profile rather than known functional similarity, the clustered modules were given the names of random colors where grey is the only module that has any pre-existing definition of containing genes that do not cluster well with others. These de-novo clustered genes were then tested for functional enrichment of known GO terms with hypergeometric tests available in the R/Bioconductor package clusterProfiler ${ }^{12}$. Significant terms with Benjamini-Hochberg adjusted p-values less than 0.05 were then collapsed by similarity into clusterProfiler category network plots to display the most significant terms for each module of hub genes in order to interpolate the function of each significant module. The information for all clustered genes for each module was then combined with their respective statistical significance results from Limma to determine whether or not those features were also found to be significantly differentially expressed.

\section{CSF1R antibody injections}

858 BioXCell) or Rat IgG (cat \#14131, Sigma) i.p. every three weeks for a total of four 859 injections. Depletion of macrophages was confirmed by CD68 immunofluorescence in the sciatic nerve.

\section{Macrophage activation assay}

Peritoneal macrophages were harvested from individual mice by peritoneal lavage using ice-cold DMEM containing 1\% FBS. Cells were then transferred into plates and subjected to either PBS or LPS treatment at a concentration of $10 \mathrm{ng} / \mathrm{mL}$ for 4 hours at $37^{\circ} \mathrm{C}$. 


\section{Statistical analysis}

869 (SEM). Between group comparisons were made with one-way and two-way ANOVA

870 with post hoc Holm-Sidak multiple comparison test or paired and unpaired t-tests as

871 appropriate. Two-sided significance tests were used throughout and $\mathrm{P}<0.05$ was

872 considered statistically significant. All statistics were calculated with the aid of Prism 9

873 (GraphPad) software.

874

875

876 
877 Acknowledgments: We thank the patients and their family for participating in our research study. We thank Milbrandt and DiAntonio labs members for helpful discussions and Cassidy Menendez, Rachel McClarney, Timothy Fahrner, and Alicia Neiner for technical support. We also thank the CHOP Genetic Counseling Core and the Genome Engineering and iPSC Center (GEIC).

Author Contributions: C.B.D., A.J.B., A.D., and J.M. conceived the overall study. All authors contributed to the study design. C.B.D. and A.S. collected and analyzed all mouse behavior data and mouse tissue samples. J.Z. performed in vitro biochemical experiments. S.W.Y. provided and interpreted clinical and genetic information. R.E.S. performed ultrastructural electron microscopy nerve analysis. P.L.W. and C.B.D. performed and analyzed flow cytometry experiments. Y.Y. performed NMJ analysis. A.Y.K. analyzed bulk RNA-Seq data. Y.S. provided early intellectual input. C.B.D. wrote the manuscript and prepared all figures. A.J.B., A.D., and J.M. oversaw the analysis and revised the manuscript. All authors gave final approval of the manuscript.

Corresponding authors: Correspondence to J.M. and A.D.

Funding: This work was supported by National Institutes of Health grants (R01NS119812 to A.J.B., A.D. and J.M, R01NS087632 to A.D. and J.M., R37NS065053 to A.D., and RF1AG013730 to J.M.)

Competing Interests: A.D. and J.M. are co-founders, scientific advisory board members, and shareholders of Disarm Therapeutics, a wholly-owned subsidiary of Eli Lilly. A.J.B. and Y.S. are consultants to Disarm Therapeutics. The authors have no other competing 


\section{REFERENCES}

909 1. Figley, M. D. \& DiAntonio, A. The SARM1 axon degeneration pathway: control of the NAD+ metabolome regulates axon survival in health and disease. Curr. Opin. Neurobiol. 63, 59-66 (2020).

2. Coleman, M. P. \& Höke, A. Programmed axon degeneration: from mouse to mechanism to medicine. Nat. Rev. Neurosci. 21, 183-196 (2020).

3. Krauss, R., Bosanac, T., Devraj, R., Engber, T. \& Hughes, R. O. Axons Matter: The Promise of Treating Neurodegenerative Disorders by Targeting SARM1-Mediated Axonal Degeneration. Trends Pharmacol. Sci. 41, 281-293 (2020).

4. Gerdts, J., Brace, E. J., Sasaki, Y., Diantonio, A. \& Milbrandt, J. Supplementary Materials for SARM1 activation triggers axon degeneration locally via NAD + destruction. Science 348, 453-458 (2015).

5. Osterloh, J. M. et al. dSarm/Sarm1 is required for activation of an injury-induced axon death pathway. Science 337, 481-484 (2012).

6. Conforti, L. et al. A Ufd2/D4Cole1e chimeric protein and overexpression of Rbp7 in the slow Wallerian degeneration (WIdS) mouse. Proc. Natl. Acad. Sci. U. S. A. 97, 11377-11382 (2000).

7. Yan, T. et al. Nmnat2 delays axon degeneration in superior cervical ganglia dependent on its NAD synthesis activity. Neurochem. Int. 56, 101-106 (2010).

8. Gilley, J. \& Coleman, M. P. Endogenous Nmnat2 is an essential survival factor for maintenance of healthy axons. PLoS Biol. 8, e1000300 (2010).

9. Figley, M. D. et al. SARM1 is a metabolic sensor activated by an increased NMN/NAD+ ratio to trigger axon degeneration. Neuron 109, 1118-1136.e11 (2021).

10. Jiang, Y. et al. The NAD+-mediated self-inhibition mechanism of proneurodegenerative SARM1. Nature 588, 658-663 (2020).

11. Sporny, M. et al. Structural basis for SARM1 inhibition and activation under energetic stress. Elife 9, (2020).

12. Gilley, J., Ribchester, R. R. \& Coleman, M. P. Sarm1 Deletion, but Not WIdS, Confers Lifelong Rescue in a Mouse Model of Severe Axonopathy. Cell Rep. 21, 10-16 (2017).

13. Gerdts, J., Summers, D. W., Sasaki, Y., DiAntonio, A. \& Milbrandt, J. Sarm1Mediated Axon Degeneration Requires Both SAM and TIR Interactions. Journal of Neuroscience 33, 13569-13580 (2013).

14. Geisler, S. et al. Prevention of vincristine-induced peripheral neuropathy by genetic deletion of SARM1 in mice. Brain 139, 3092-3108 (2016).

15. Henninger, N. et al. Attenuated traumatic axonal injury and improved functional outcome after traumatic brain injury in mice lacking Sarm1. Brain 139, 1094-1105 (2016).

16. Turkiew, E., Falconer, D., Reed, N. \& Höke, A. Deletion of Sarm1 gene is neuroprotective in two models of peripheral neuropathy. J. Peripher. Nerv. Syst. 22, 162-171 (2017).

17. Yin, T. C. et al. Acute Axonal Degeneration Drives Development of Cognitive, Motor, and Visual Deficits after Blast-Mediated Traumatic Brain Injury in Mice. eNeuro 3, (2016).

18. Ziogas, N. K. \& Koliatsos, V. E. Primary Traumatic Axonopathy in Mice Subjected to Impact Acceleration: A Reappraisal of Pathology and Mechanisms with HighResolution Anatomical Methods. J. Neurosci. 38, 4031-4047 (2018).

19. Ferri, A., Sanes, J. R., Coleman, M. P., Cunningham, J. M. \& Kato, A. C. Inhibiting axon degeneration and synapse loss attenuates apoptosis and disease progression 
in a mouse model of motoneuron disease. Curr. Biol. 13, 669-673 (2003).

20. Howell, G. R., Soto, I., Libby, R. T. \& John, S. W. M. Intrinsic axonal degeneration pathways are critical for glaucomatous damage. Exp. Neurol. 246, 54-61 (2013).

21. Williams, P. A. et al. Nicotinamide and WLDS Act Together to Prevent Neurodegeneration in Glaucoma. Front. Neurosci. 11, 232 (2017).

22. Geisler, S. et al. Gene therapy targeting SARM1 blocks pathological axon degeneration in mice. J. Exp. Med. 216, jem.20181040 (2019).

23. Wang, M. S., Davis, A. A., Culver, D. G. \& Glass, J. D. WIdS mice are resistant to paclitaxel (taxol) neuropathy. Ann. Neurol. 52, 442-447 (2002).

24. Zhu, S. S. et al. Wld S protects against peripheral neuropathy and retinopathy in an experimental model of diabetes in mice. Diabetologia 54, 2440 (2011).

25. Ko, K. W., Milbrandt, J. \& DiAntonio, A. SARM1 acts downstream of neuroinflammatory and necroptotic signaling to induce axon degeneration. J. Cell Biol. 219, (2020).

26. Sasaki, Y. et al. SARM1 depletion rescues NMNAT1-dependent photoreceptor cell death and retinal degeneration. Elife 9, (2020).

27. Huppke, P. et al. Homozygous NMNAT2 mutation in sisters with polyneuropathy and erythromelalgia. Exp. Neurol. 112958 (2019) doi:10.1016/J.EXPNEUROL.2019.112958.

28. Lukacs, M. et al. Severe biallelic loss-of-function mutations in nicotinamide mononucleotide adenylyltransferase 2 (NMNAT2) in two fetuses with fetal akinesia deformation sequence. Exp. Neurol. 112961 (2019) doi:10.1016/J.EXPNEUROL.2019.112961.

29. Joseph Bloom, A. et al. Constitutively active SARM1 variants found in ALS patients induce neuropathy. bioRxiv 2021.04.16.439886 (2021) doi:10.1101/2021.04.16.439886.

30. Gilley, J. et al. Enrichment of SARM1 alleles encoding variants with constitutively hyperactive NADase in patients with ALS and other motor nerve disorders. medRxiv (2021).

31. Sasaki, Y. et al. cADPR is a gene dosage-sensitive biomarker of SARM1 activity in healthy, compromised, and degenerating axons. Exp. Neurol. 329, 113252 (2020).

32. Zigmond, R. E. \& Echevarria, F. D. Macrophage biology in the peripheral nervous system after injury. Prog. Neurobiol. 173, 102-121 (2019).

33. Klein, D. \& Martini, R. Myelin and macrophages in the PNS: An intimate relationship in trauma and disease. Brain Res. 1641, 130-138 (2016).

34. Shepherd, A. J. et al. Macrophage angiotensin II type 2 receptor triggers neuropathic pain. Proc. Natl. Acad. Sci. U. S. A. 115, E8057-E8066 (2018).

35. Cattin, A.-L. et al. Macrophage-Induced Blood Vessels Guide Schwann CellMediated Regeneration of Peripheral Nerves. Cell 162, 1127-1139 (2015).

36. Barrette, B. et al. Requirement of myeloid cells for axon regeneration. J. Neurosci. 28, 9363-9376 (2008).

37. Brück, W., Huitinga, I. \& Dijkstra, C. D. Liposome-mediated monocyte depletion during wallerian degeneration defines the role of hematogenous phagocytes in myelin removal. J. Neurosci. Res. 46, 477-484 (1996).

38. Dailey, A. T., Avellino, A. M., Benthem, L., Silver, J. \& Kliot, M. Complement depletion reduces macrophage infiltration and activation during Wallerian degeneration and axonal regeneration. J. Neurosci. 18, 6713-6722 (1998).

39. Liu, T., van Rooijen, N. \& Tracey, D. J. Depletion of macrophages reduces axonal degeneration and hyperalgesia following nerve injury. Pain 86, 25-32 (2000).

40. Jafari, M. et al. Phagocyte-mediated synapse removal in cortical neuroinflammation 
is promoted by local calcium accumulation. Nat. Neurosci. 24, 355-367 (2021).

41. Starobova, H. et al. Vincristine-induced peripheral neuropathy is driven by canonical NLRP3 activation and IL-1ß release. J. Exp. Med. 218, (2021).

42. Graykowski, D. \& Cudaback, E. Don't know what you got till it's gone: microglial depletion and neurodegeneration. Neural Regeneration Res. 16, 1921-1927 (2021).

43. Fleckenstein, J. L. et al. Denervated human skeletal muscle: MR imaging evaluation. Radiology 187, 213-218 (1993).

44. Schmidt, T. G. M. \& Skerra, A. The Strep-tag system for one-step purification and high-affinity detection or capturing of proteins. Nat. Protoc. 2, 1528-1535 (2007).

45. Fabry, V. et al. Which Method for Diagnosing Small Fiber Neuropathy? Front. Neurol. 11, 342 (2020).

46. de la Motte, D. J., Hall, S. M. \& Allt, G. A study of the perineurium in peripheral nerve pathology. Acta Neuropathol. 33, 257-270 (1975).

47. Schaefer, A. M., Sanes, J. R. \& Lichtman, J. W. A compensatory subpopulation of motor neurons in a mouse model of amyotrophic lateral sclerosis. J. Comp. Neurol. 490, 209-219 (2005).

48. Ang, E.-T. et al. Motor axonal sprouting and neuromuscular junction loss in an animal model of Charcot-Marie-Tooth disease. J. Neuropathol. Exp. Neurol. 69, 281-293 (2010).

49. Yin, X. et al. Dysmyelinated Lower Motor Neurons Retract and Regenerate Dysfunctional Synaptic Terminals. J. Neurosci. 24, 3890-3898 (2004).

50. Gale, A. N., Gomez, S. \& Duchen, L. W. Changes produced by a hypomyelinating neuropathy in muscle and its innervation. Morphological and physiological studies in the Trembler mouse. Brain 105, 373-393 (1982).

51. Hughes, R. O. et al. Small Molecule SARM1 Inhibitors Recapitulate the SARM1-/Phenotype and Allow Recovery of a Metastable Pool of Axons Fated to Degenerate. Cell Rep. 34, 108588 (2021).

52. Geisler, S. et al. Vincristine and bortezomib use distinct upstream mechanisms to activate a common SARM1-dependent axon degeneration program. JCI Insight 4, (2019).

53. Jakovčevski, I., Förster, E., Reiss, G. \& Schachner, M. Impact of Depletion of Microglia/Macrophages on Regeneration after Spinal Cord Injury. Neuroscience 459, 129-141 (2021).

54. Rios, R., Jablonka-Shariff, A., Broberg, C. \& Snyder-Warwick, A. K. Macrophage roles in peripheral nervous system injury and pathology: Allies in neuromuscular junction recovery. Mol. Cell. Neurosci. 111, 103590 (2021).

55. Koike, H. \& Katsuno, M. The role of macrophages in Guillain-Barré syndrome and chronic inflammatory demyelinating polyneuropathy. Neurol. Clin. Neurosci. (2021) doi:10.1111/ncn3.12496.

56. Silva, C. E. A., Guimarães, R. M. \& Cunha, T. M. Sensory neuron-associated macrophages as novel modulators of neuropathic pain. Pain Rep 6, e873 (2021).

57. Bartels, T., De Schepper, S. \& Hong, S. Microglia modulate neurodegeneration in Alzheimer's and Parkinson's diseases. Science 370, 66-69 (2020).

58. Devanney, N. A., Stewart, A. N. \& Gensel, J. C. Microglia and macrophage metabolism in CNS injury and disease: The role of immunometabolism in neurodegeneration and neurotrauma. Exp. Neurol. 329, 113310 (2020).

59. Mammana, S. et al. The Role of Macrophages in Neuroinflammatory and Neurodegenerative Pathways of Alzheimer's Disease, Amyotrophic Lateral Sclerosis, and Multiple Sclerosis: Pathogenetic Cellular Effectors and Potential 
Therapeutic Targets. Int. J. Mol. Sci. 19, (2018).

60. Shi, Y. et al. Microglia drive APOE-dependent neurodegeneration in a tauopathy mouse model. J. Exp. Med. 216, 2546-2561 (2019).

61. Minhas, P. S. et al. Macrophage de novo NAD+ synthesis specifies immune function in aging and inflammation. Nat. Immunol. 20, 50-63 (2019).

62. Wang, P. L. et al. Peripheral nerve resident macrophages share tissue-specific programming and features of activated microglia. Nat. Commun. 11, 2552 (2020).

63. Lavin, Y. et al. Tissue-resident macrophage enhancer landscapes are shaped by the local microenvironment. Cell 159, 1312-1326 (2014).

64. Chakarov, S. et al. Two distinct interstitial macrophage populations coexist across tissues in specific subtissular niches. Science 363, (2019).

65. Bennett, F. C. et al. A Combination of Ontogeny and CNS Environment Establishes Microglial Identity. Neuron 98, 1170-1183.e8 (2018).

66. Van Hove, $\mathrm{H}$. et al. A single-cell atlas of mouse brain macrophages reveals unique transcriptional identities shaped by ontogeny and tissue environment. Nat.

Neurosci. 22, 1021-1035 (2019).

67. Clements, M. P. et al. The Wound Microenvironment Reprograms Schwann Cells to Invasive Mesenchymal-like Cells to Drive Peripheral Nerve Regeneration. Neuron 96, 98-114.e7 (2017).

68. Painter, M. W. et al. Diminished Schwann cell repair responses underlie ageassociated impaired axonal regeneration. Neuron 83, 331-343 (2014).

69. Jessen, K. R. \& Mirsky, R. The repair Schwann cell and its function in regenerating nerves. J. Physiol. 594, 3521-3531 (2016).

70. Yim, A. K. Y. et al. Disentangling glial diversity in peripheral nerves at single-nuclei resolution. Nat. Neurosci. (2022) doi:10.1038/s41593-021-01005-1.

71. Stanley, E. R. \& Chitu, V. CSF-1 receptor signaling in myeloid cells. Cold Spring Harb. Perspect. Biol. 6, (2014).

72. MacDonald, K. P. A. et al. An antibody against the colony-stimulating factor 1 receptor depletes the resident subset of monocytes and tissue- and tumorassociated macrophages but does not inhibit inflammation. Blood 116, 3955-3963 (2010).

73. Gilley, J., Mayer, P. R., Yu, G. \& Coleman, M. P. Low levels of NMNAT2 compromise axon development and survival. Hum. Mol. Genet. 28, 448-458 (2019).

74. Gilley, J., Orsomando, G., Nascimento-Ferreira, I. \& Coleman, M. P. Absence of SARM1 Rescues Development and Survival of NMNAT2-Deficient Axons. Cell Rep. 10, 1974-1981 (2015).

75. Brioschi, S., Zhou, Y. \& Colonna, M. Brain Parenchymal and Extraparenchymal Macrophages in Development, Homeostasis, and Disease. J. Immunol. 204, 294305 (2020).

76. Minett, T. et al. Microglial immunophenotype in dementia with Alzheimer's pathology. J. Neuroinflammation 13, 135 (2016).

77. Bodea, L.-G. et al. Neurodegeneration by activation of the microglial complementphagosome pathway. J. Neurosci. 34, 8546-8556 (2014).

78. Züchner, S. \& Vance, J. M. Mechanisms of disease: a molecular genetic update on hereditary axonal neuropathies. Nat. Clin. Pract. Neurol. 2, 45-53 (2006).

79. Marshall, K. L. \& Farah, M. H. Axonal regeneration and sprouting as a potential therapeutic target for nervous system disorders. Neural Regeneration Res. 16, 1901-1910 (2021).

80. Muzio, L., Viotti, A. \& Martino, G. Microglia in Neuroinflammation and 

(2021).

81. Marion, C. M., McDaniel, D. P. \& Armstrong, R. C. Sarm1 deletion reduces axon damage, demyelination, and white matter atrophy after experimental traumatic brain injury. Exp. Neurol. 321, 113040 (2019).

82. Ozaki, E. et al. SARM1 deficiency promotes rod and cone photoreceptor cell survival in a model of retinal degeneration. Life Sci Alliance 3, (2020).

83. Gibson, K. M. et al. Novel findings with reassessment of exome data: implications for validation testing and interpretation of genomic data. Genet. Med. 20, 329-336 (2018).

84. Sasaki, Y., Hackett, A. R., Kim, S., Strickland, A. \& Milbrandt, J. Dysregulation of $\mathrm{NAD}+$ Metabolism Induces a Schwann Cell Dedifferentiation Program. J. Neurosci. 38, 6546-6562 (2018).

85. Hackett, A. R., Strickland, A. \& Milbrandt, J. Disrupting insulin signaling in Schwann cells impairs myelination and induces a sensory neuropathy. Glia 68, 963-978 (2020).

86. Beirowski, B. et al. Sir-two-homolog 2 (Sirt2) modulates peripheral myelination through polarity protein Par-3/atypical protein kinase C (aPKC) signaling. Proc. Natl. Acad. Sci. U. S. A. 108, E952-61 (2011).

87. Schildhaus, N. et al. Thermal latency studies in opiate-treated mice. J. Pharm. Bioallied Sci. 6, 43-47 (2014).

88. Deacon, R. M. J. Measuring the strength of mice. J. Vis. Exp. (2013) doi:10.3791/2610. 

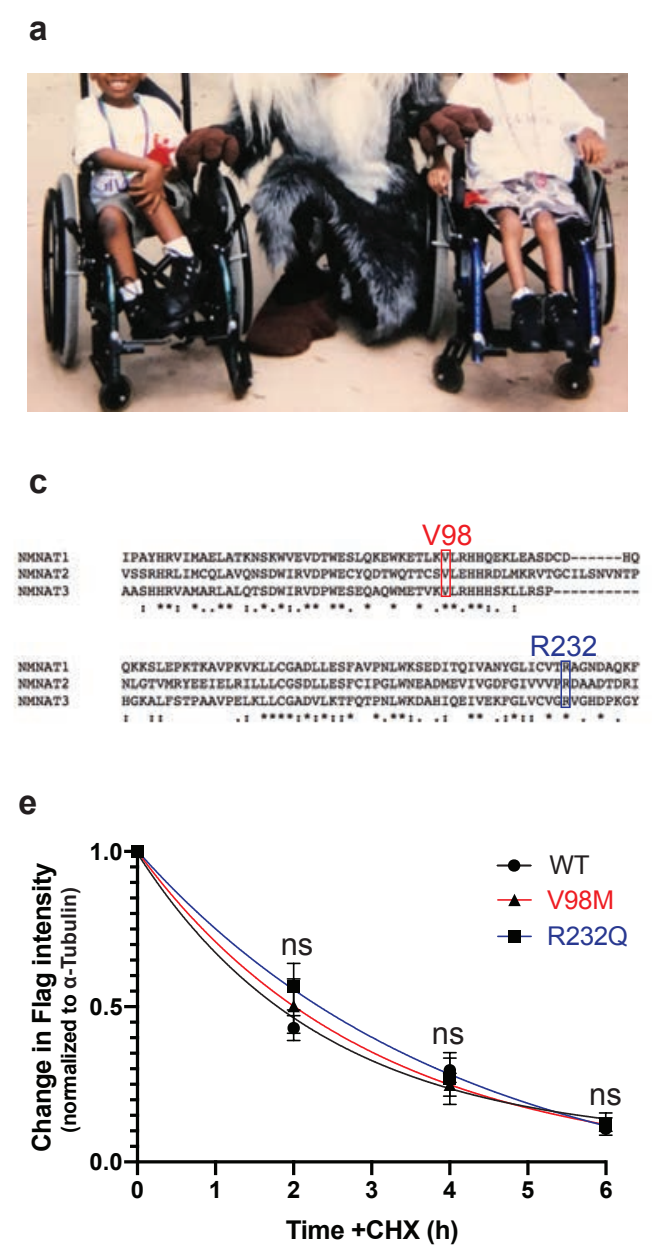

b
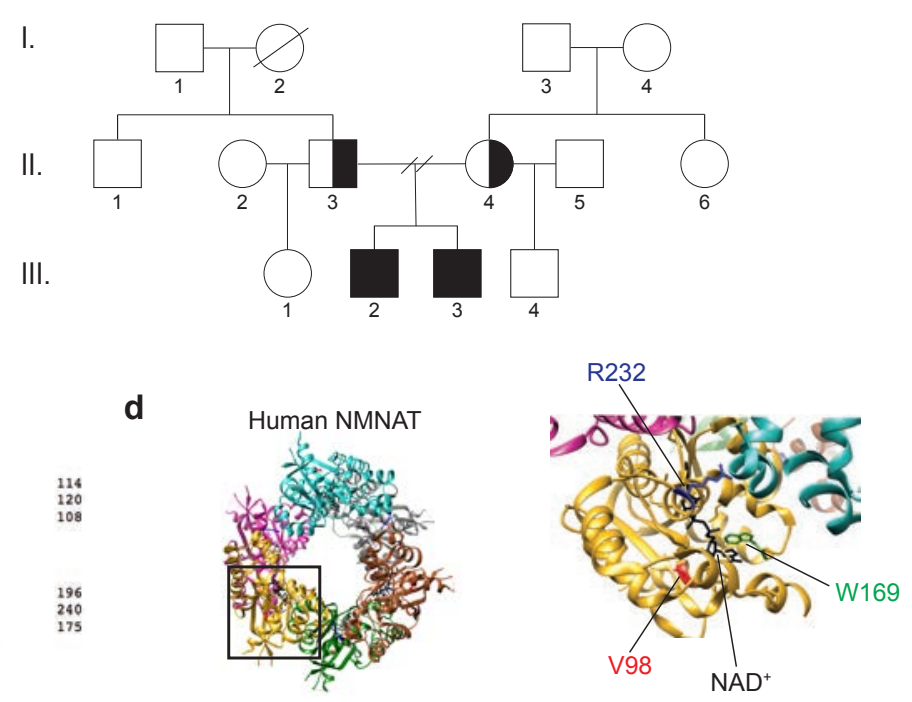

f

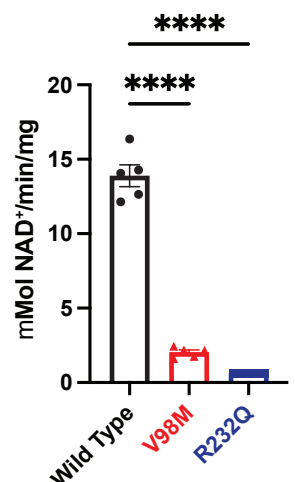

1131
Figure 1: Identification of compound heterozygous NMNAT2 variants in two brothers with relapsing-remitting neuropathy. a, Clinical features of two brothers suffering a unique sensorimotor neuropathy syndrome. b, WES showed that both brothers carry two extremely rare missense mutations in the NMNAT2 gene (c.292G>A. c.695G $>A$ ), each inherited from one of their parents. c, Both residues are conserved in all three human NMNAT isoforms. d, Schematic of NMNAT structure. The location of the patients' missense variants is noted in red (V98) and blue (R232). W169 (green) is the catalytic residue. e, Relative turnover rates for Flag-NMNAT2 (wild-type, V98M, R232Q) after cycloheximide (CHX) addition. Changes in Flag-NMNAT intensity were normalized to $\alpha$-tubulin (loading control) and calculated as a proportion of the intensity of the $0 \mathrm{~h}$ untreated band for each variant. One-phase decay curves were fitted to the data using non-linear regression. $\mathbf{f}$, NMNAT activity assay was performed on purified NMNAT2 at $37^{\circ} \mathrm{C}$. NAD ${ }^{+}$production at steady state $(10 \mathrm{~min})$ was used to calculate the NMNAT activity. All data are presented as mean $+/$ - SEM from $n=5$ independent experiments. Statistical significance was determined by two-way ANOVA with multiple comparisons. ns: not significant, ${ }^{* * *} p<0.0001$. 
a

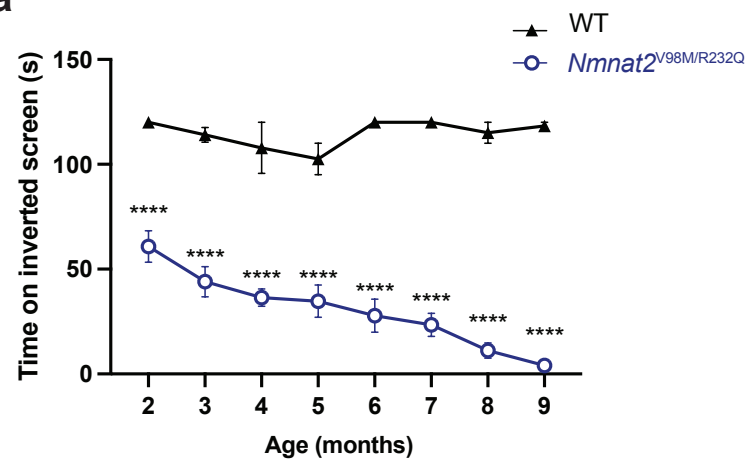

C
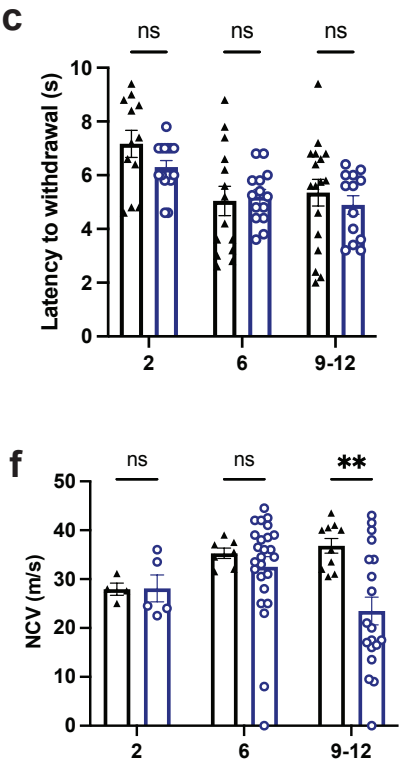

d

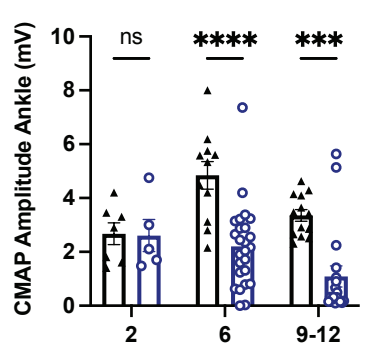

g

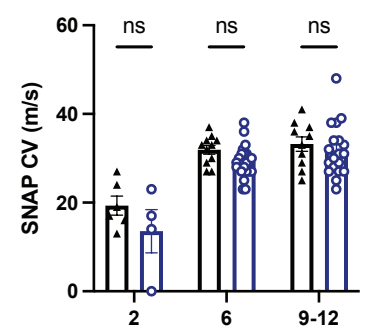

b

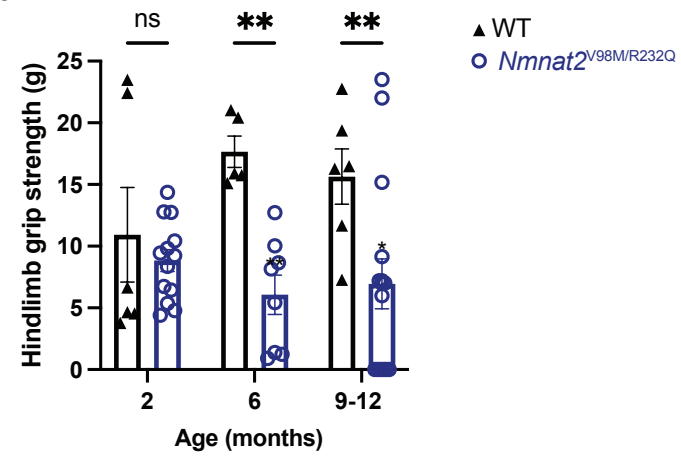

e
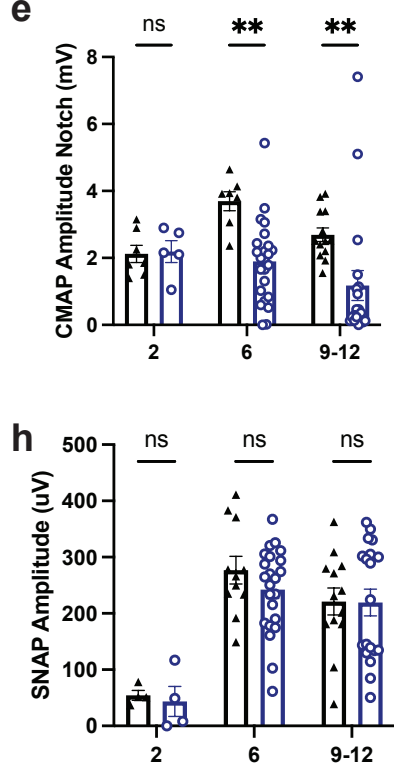

Figure 2: Nmnat2 ${ }^{V 98 M / R 232 Q}$ mice have behavioral and electrophysiologic features consistent with a motor neuropathy. a, Average time suspended from an inverted screen (max. 120s) for WT or Nmnat2 ${ }^{\mathrm{V} 98 \mathrm{M} / \mathrm{R} 232 \mathrm{Q}}$ mice. b, Hindlimb grip strength for WT or Nmnat2 ${ }^{V 98 M / R 232 Q}$ mice at 2, 6, and 9-12 months of age. c, The average time it takes for WT or Nmnat2 $2^{V 98 M / R 232 Q}$ mice to remove their tails from a $55^{\circ} \mathrm{C}$ hot water bath at 2, 6 , and 9-12 months of age. d-e, CMAP amplitude of WT and Nmnat $2^{V 98 M / R 232 Q}$ mice at the ankle (d) and sciatic notch (e) at 2, 6, and 9-12 months of age. f, NCV (m/s) of sciatic nerves of WT or Nmnat $2^{V 98 M / R 232 Q}$ mice at 2, 6, and 9-12 months of age. g, SNAP CV $(\mathrm{m} / \mathrm{s})$ of sciatic nerves of WT or Nmnat2 ${ }^{V 98 M / R 232 Q}$ mice at 2, 6, and 9-12 months of age. $\mathbf{h}$, SNAP amplitude $(\mu \mathrm{V})$ of sciatic nerves of WT and Nmnat2 ${ }^{V 98 M / R 232 Q}$ mice at 2, 6, and 9-12 months of age. All data are presented as mean +/- SEM. Statistical significance was determined by two-way ANOVA with multiple comparisons. ns: not significant, * $p<0.05$, ${ }^{* *} p<0.01,{ }^{* * *} p<0.001,{ }^{* * * *} p<0.0001$. 


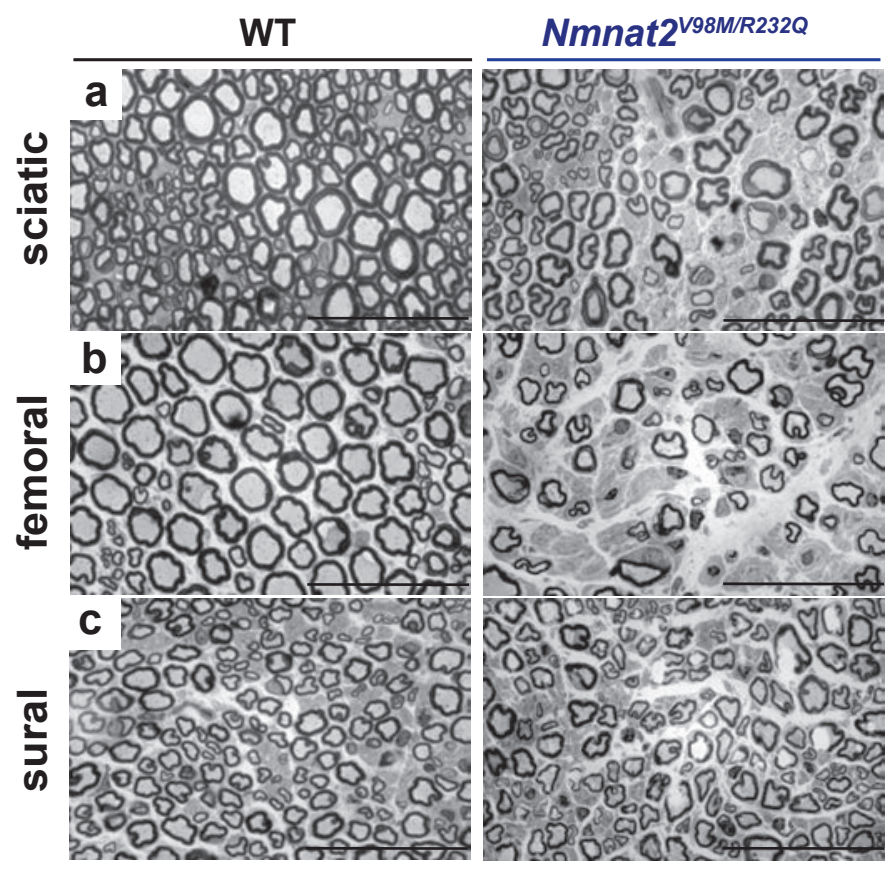

h
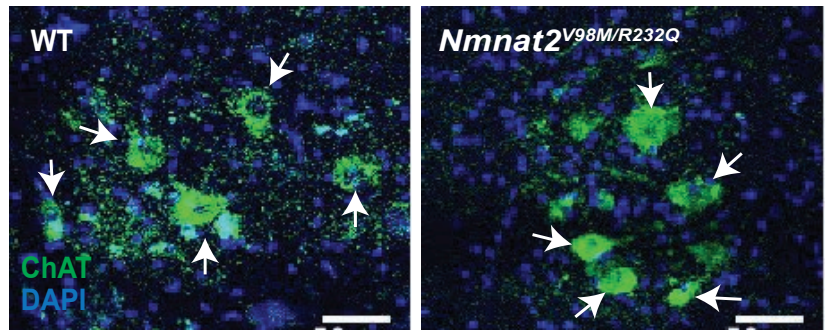

axon area/total nerve area
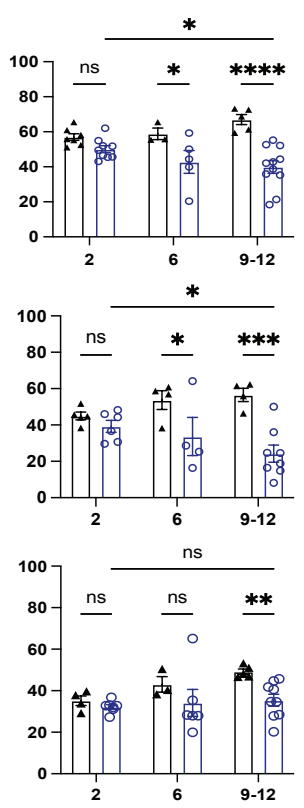

Spinal motor neuron counts (ventral horn)

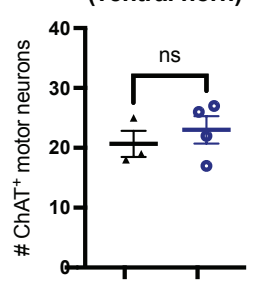

$$
\text { i }
$$
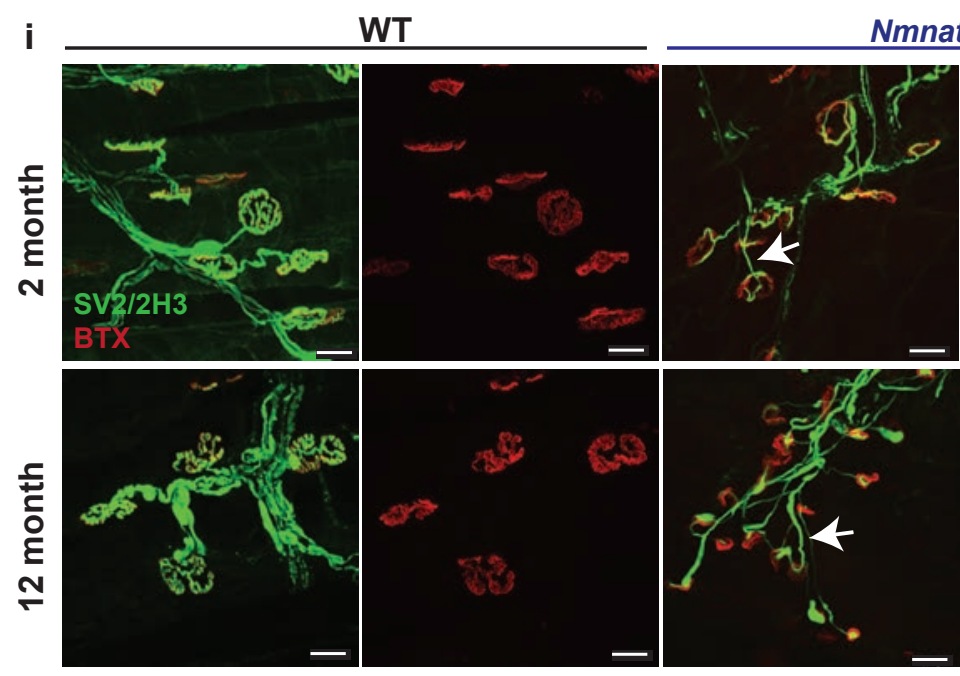

m

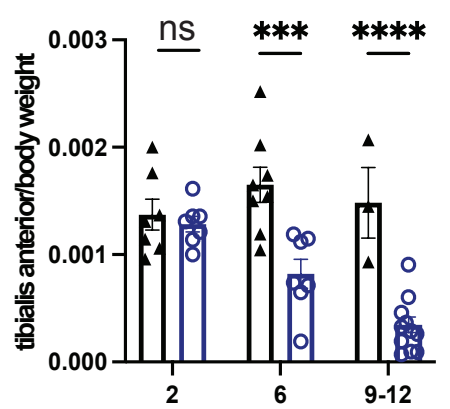

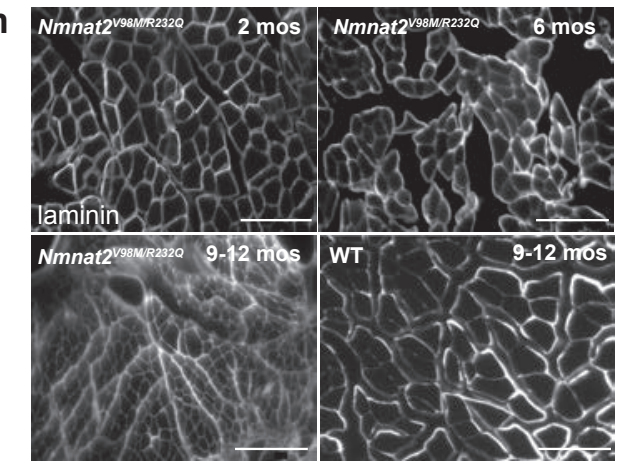

Nmnat2 ${ }^{\text {V98M/R232Q }}$

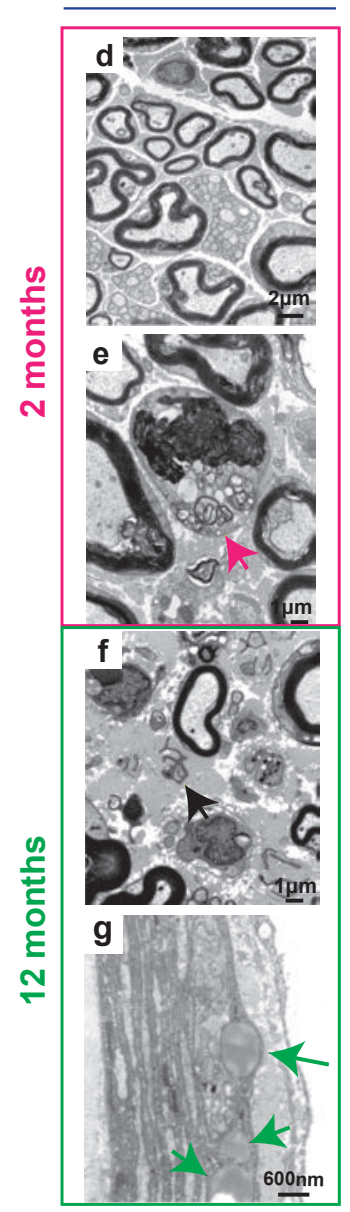

j

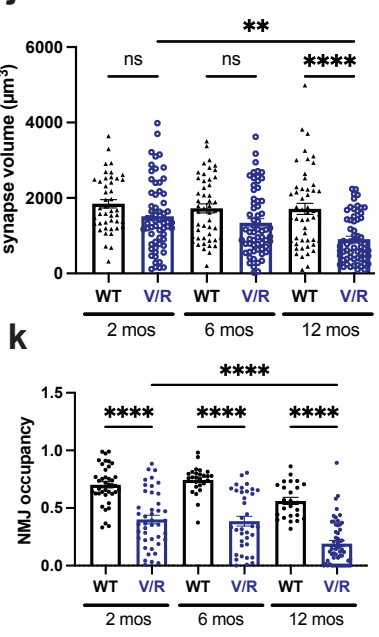

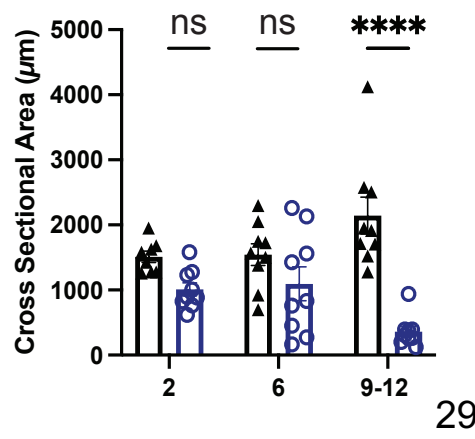


1164 Figure 3: Nmnat2 variants cause progressive axon loss and muscle wasting in mice. a-c, Representative images of sciatic (a), femoral (b), and sural (c) nerves in 9-12month-old Nmnat2 ${ }^{V 98 M / R 232 Q}$ or WT mice. Percent axon area as a ratio of total nerve area is calculated to the right. Scale bar represents $50 \mu \mathrm{m}$. d, The appearance of the sciatic nerve at two months shows a dense population of large and small myelinated axons with little intervening extracellular space. e, Macrophage containing axonal and myelin debris are found in the endoneurial space of two-month-old Nmnat2 ${ }^{V 98 M / R 232 Q}$ sciatic nerves. $\mathbf{f}$, The sciatic nerve at 12 months shows patches of marked axon loss with increased collagen and wispy processes of Schwann cells. Scattered macrophages with axonal and myelin debris are identified. $\mathbf{g}$. Presence of large perineurial droplets of neutral fat. h, Representative images of ChAT immunostaining in 12-month-old Nmnat2 ${ }^{\mathrm{V} 98 \mathrm{M} / \mathrm{R} 232 \mathrm{Q}}$ or WT mouse spinal cord (ventral horn), scale bar represents $50 \mu \mathrm{m}$. Quantification of number of $\mathrm{ChAT}^{+}$motor neuron cell bodies in the ventral horn to the right. i, Representative images of 2- and 12-month-old mouse NMJs stained for synaptic vesicle

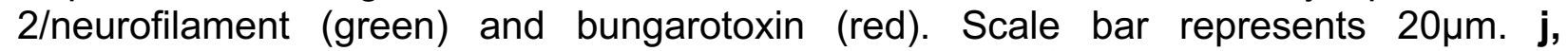
Quantification of synapse volume for WT and Nmnat2 ${ }^{V 98 M / R 232 Q}$ NMJs. k, Quantification of overlap between presynaptic area vs. postsynaptic area for WT and Nmnat2 ${ }^{\text {V98M/R232Q }}$ NMJs at 2, 6, and 12 months of age. I, Average tibialis anterior weight/body weight for WT and Nmnat2 ${ }^{V 98 M / R 232 Q}$ mice in 2, 6, and 9-12-month-old mice. $\mathrm{m}$, Representative images of laminin immunofluorescence in $N$ mnat2 ${ }^{V 98 M / R 232 Q}$ mouse tibialis anterior muscles at 2, 6, and 9-12 months of age. WT mouse tibialis anterior muscle at 12 months of age shown for comparison. The apparent fuzziness shown in the representative image of Nmnat2 ${ }^{V 98 M / R 232 Q}$ 9-12-month muscle was a consistent genotype-dependent finding reflecting diffuse laminin staining. Quantification of muscle fiber cross-sectional area to the right. Scale bar represents $150 \mu \mathrm{m}$. All data are presented as mean +/- SEM. Statistical significance was determined by Student's unpaired t-test or two-way ANOVA with multiple comparisons. ns: not significant, ${ }^{*} p<0.05,{ }^{* * *} p<0.001,{ }^{* * * *} p<0.0001$. 

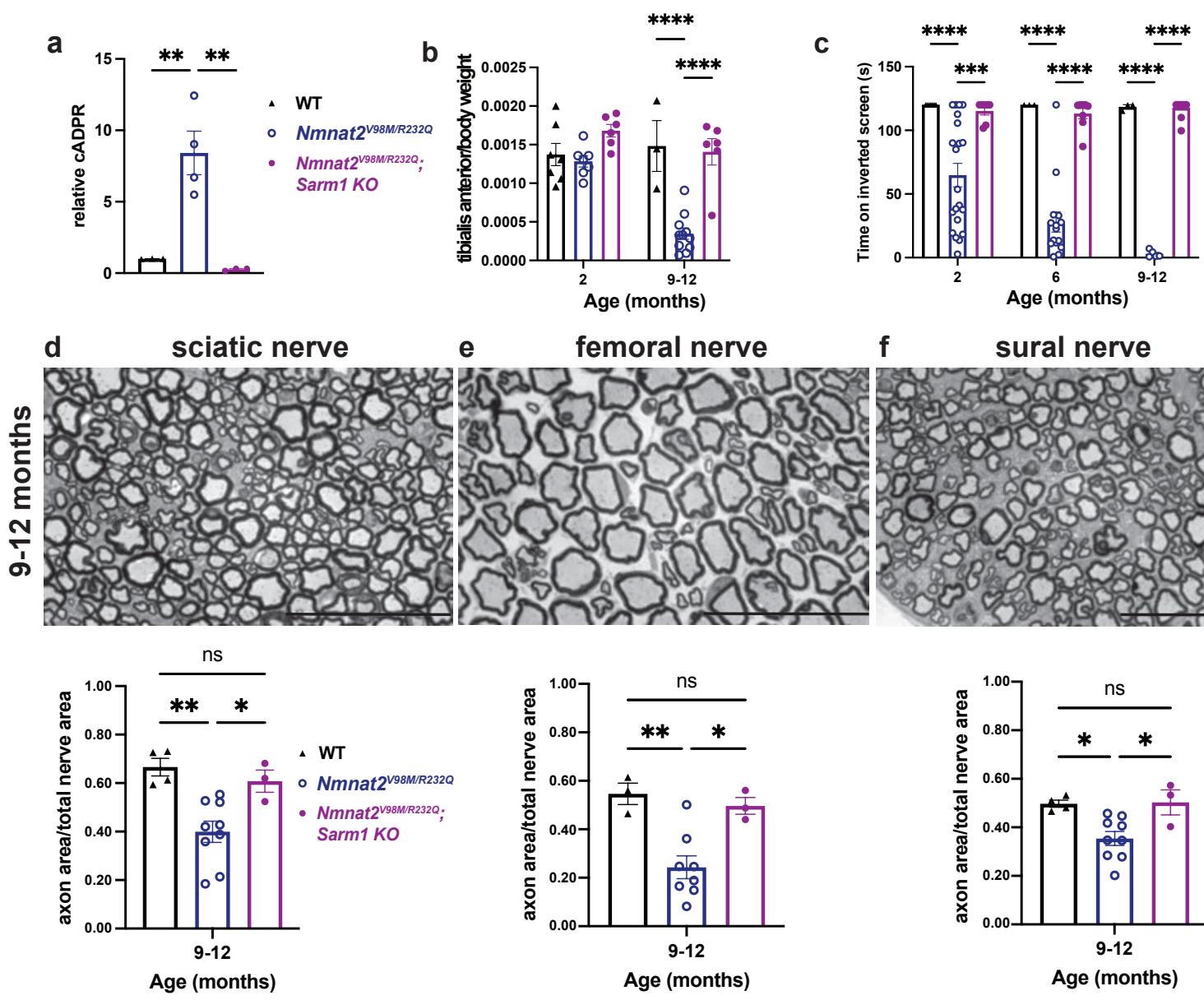

e femoral nerve

f sural nerve
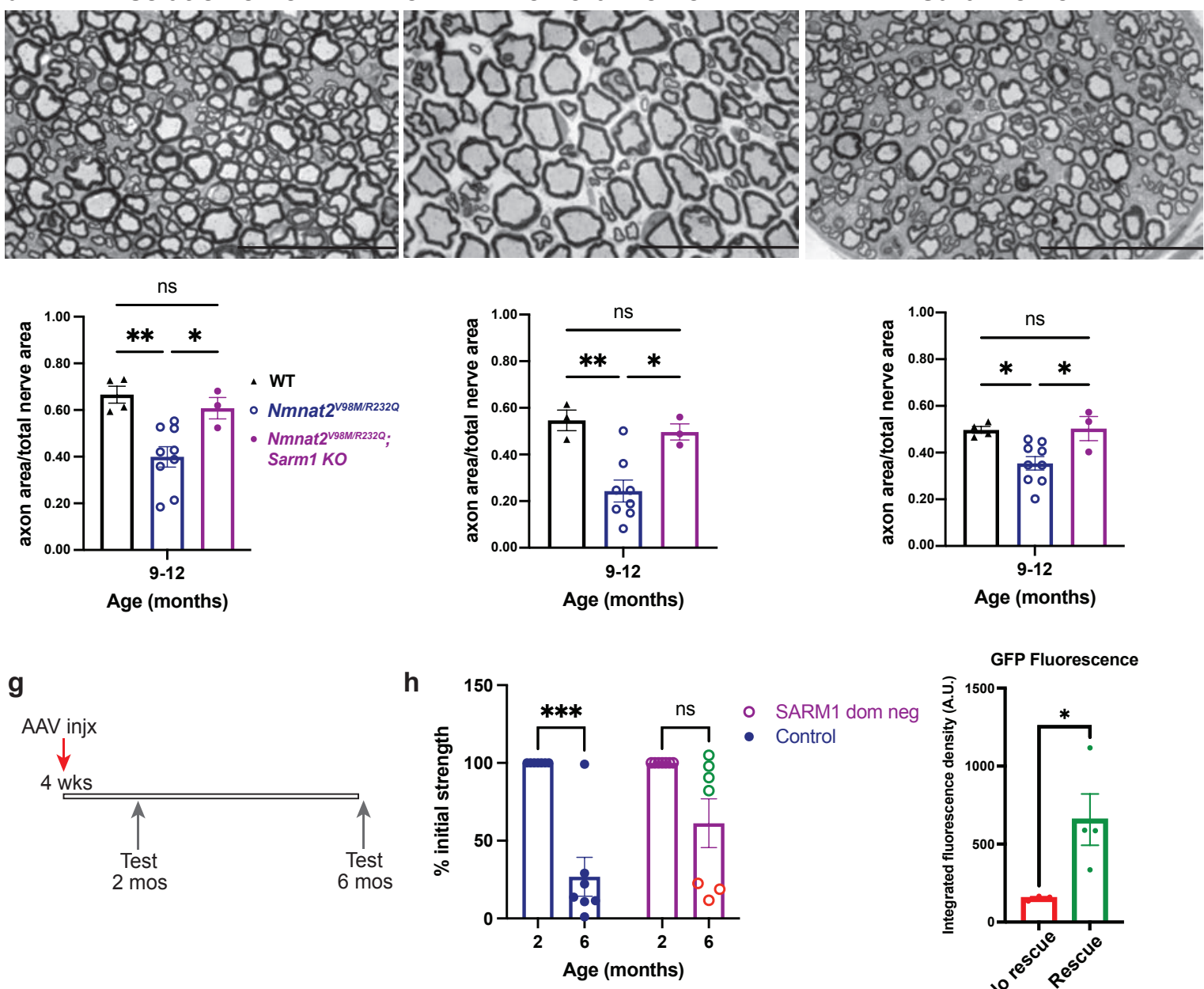

Figure 4: Sarm1 knockout rescues neuropathy caused by Nmnat2 variants in mice. a, Relative cADPR levels in sciatic nerves of 2-month-old WT, Nmnat2 ${ }^{V 98 M / R 232 Q}$, and Nmnat2 ${ }^{V 98 M / R 232 Q}$; Sarm1 KO mice. Values normalized to WT cADPR levels (set to 1). Statistical significance determined by a Student's unpaired t-test, ${ }^{* *} p<0.01$. b, Average tibialis anterior weight by body weight for WT, Nmnat2 ${ }^{V 98 M / R 232 Q}$, and Nmnat2 ${ }^{V 98 M / R 232 Q}$; Sarm $1 \mathrm{KO}$ mice in 2 and 9-12-month-old mice. c, Average time suspended from an

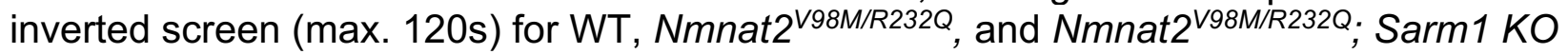
mice. d-f, Representative images of sciatic (d), femoral (e), and sural (f) nerves in 9-12month-old Nmnat2 ${ }^{V 98 M / R 232 Q}$; Sarm $1 \mathrm{KO}$ mice. Scale bar represents 50 $\mathrm{mm}$. Percent axonal area as a ratio of total nerve area is calculated below each corresponding nerve. All data are presented as mean +/- SEM. Statistical significance was determined by two-way ANOVA with multiple comparisons. ns: not significant, ${ }^{*} p<0.05,{ }^{* *} p<0.01,{ }^{* * *} p<0.001$, ${ }^{* * * *} p<0.0001$. g, Schematic of AAV-SARM1-DN gene therapy experiment. $\mathbf{h}$, Percent 
1206 (control) or SARM1 dominant-negative injected Nmnat2 ${ }^{\text {V98M/R232Q }}$ mice. Statistical 1207 significance within treatment group was determined by a Student's paired t-test, ns: not 1208 significant, ${ }^{* *} p<0.01$. Statistical significance between treatment groups was determined 1209 by a Student's unpaired t-test, ns: not significant, ${ }^{*} p<0.05$. 
bioRxiv preprint doi: https://doi.org/10.1101/2022.02.26.482110; this version posted March 1, 2022. The copyright holder for this preprint (which was not certified by peer review) is the author/funder. All rights reserved. No reuse allowed without permission.
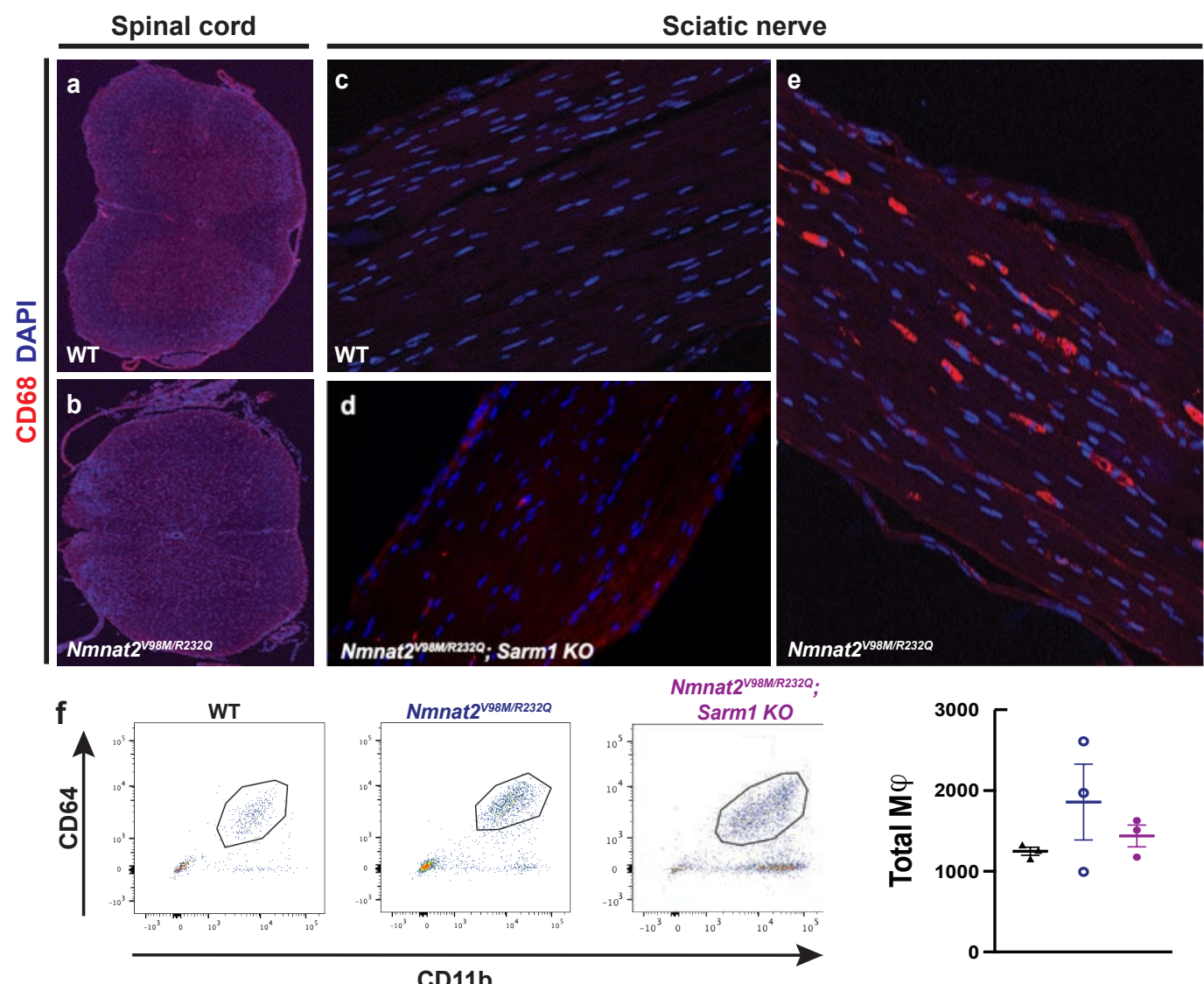

g

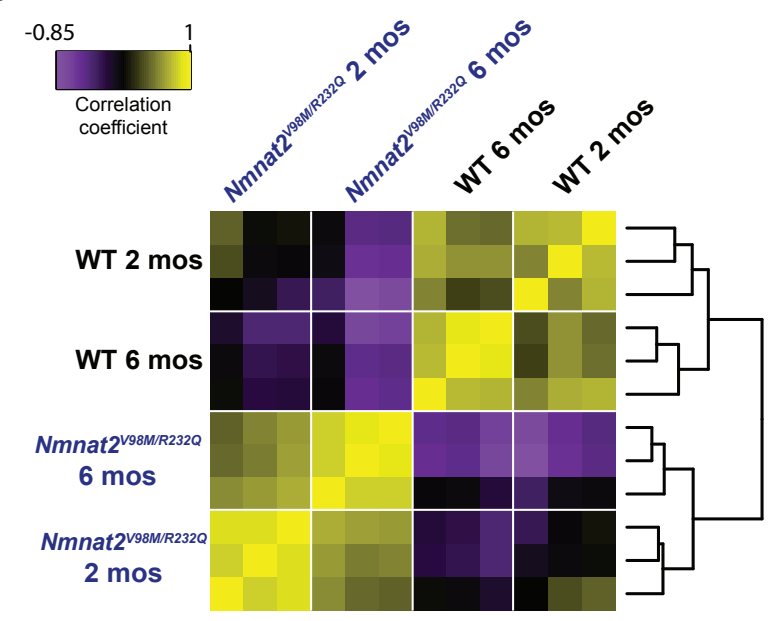

i

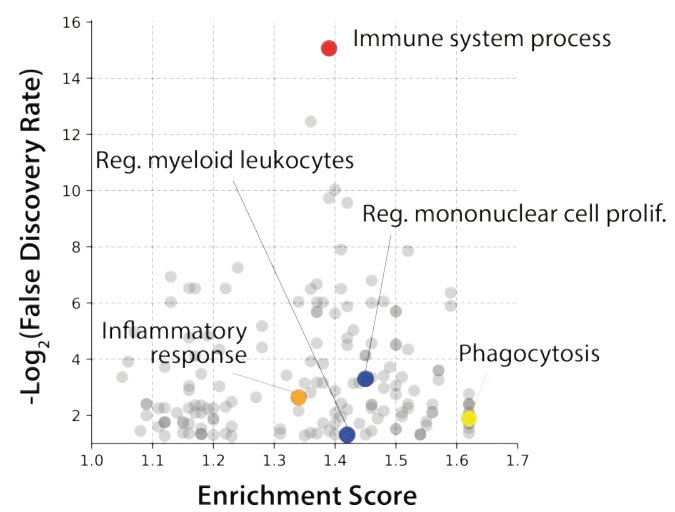

h

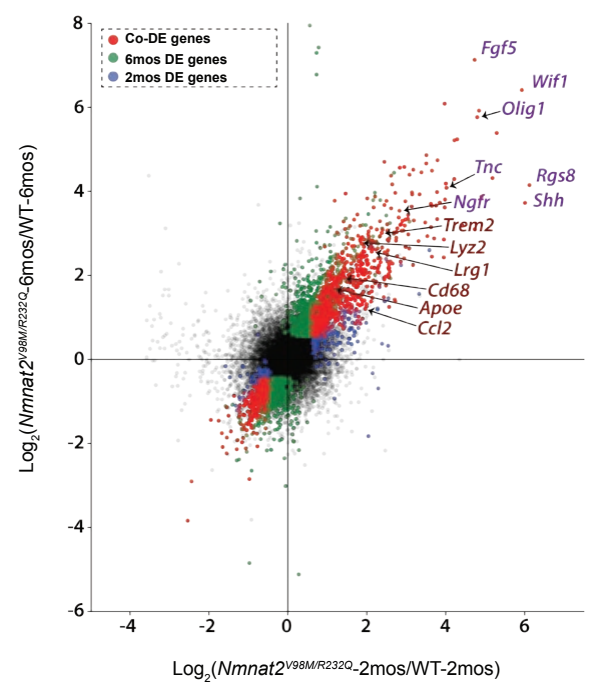


1211 Figure 5: Activated macrophages accumulate in the peripheral nervous system of Nmnat2 ${ }^{\text {V98M/R232Q }}$ mice. a-e, Representative images of CD68 immunofluorescence and DAPI signal in the spinal cord (a-b) and sciatic nerves (c-e) of 2-month-old WT, Nmnat2 , Sarm1 KO mice. f, Representative scatter plots and quantification of fluorescence-activated cell sorting of total sciatic nerve macrophages $\left(\mathrm{CD}^{+} 4^{+} \mathrm{CD} 1 \mathrm{~b}^{+}\right)$in 2-month-old $\mathrm{WT}, \quad N$ mat2 ${ }^{\mathrm{V} 98 M / R 232 \mathrm{Q}}$, and Nmnat2V98M/R232Q; Sarm1 KO mice. g, Sample correlation plot showing global transcriptomic analysis and hierarchical clustering of sciatic nerve macrophages from WT and Nmnat2 ${ }^{V 98 M / R 232 Q}$ mice. Each box represents one replicate. $\mathbf{h}$, Volcano plot of significant co-differentially expressed genes (DEGs) in 2-month and 6-month Nmnat2 ${ }^{V 98 M / R 232 Q}$ sciatic nerves highlighting activated macrophage markers and repair Schwann cell signatures. i, GO analysis of genes enriched in 6-month Nmnat2 ${ }^{\text {V98M/R232Q }}$ sciatic nerves. 
a

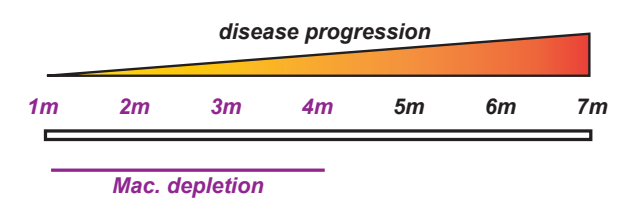

c
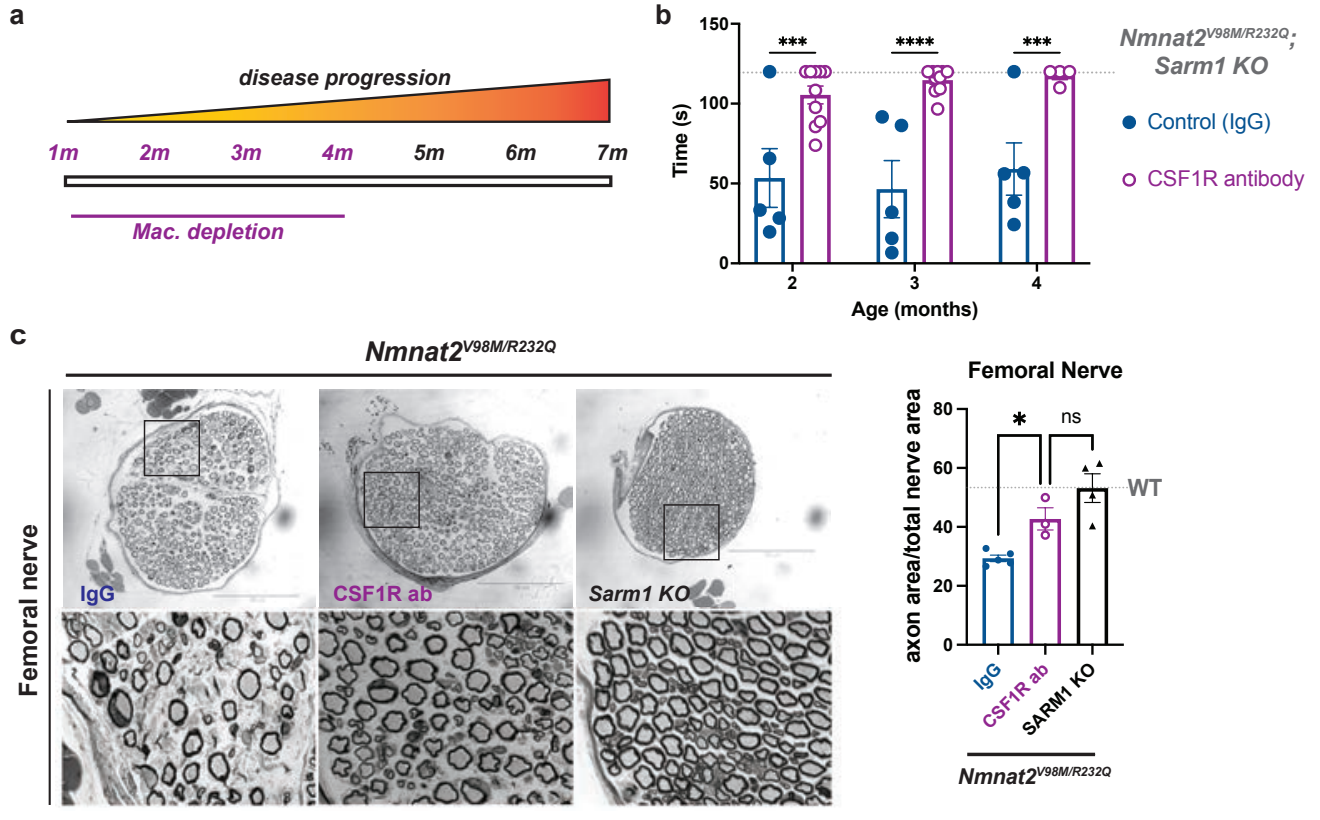

d
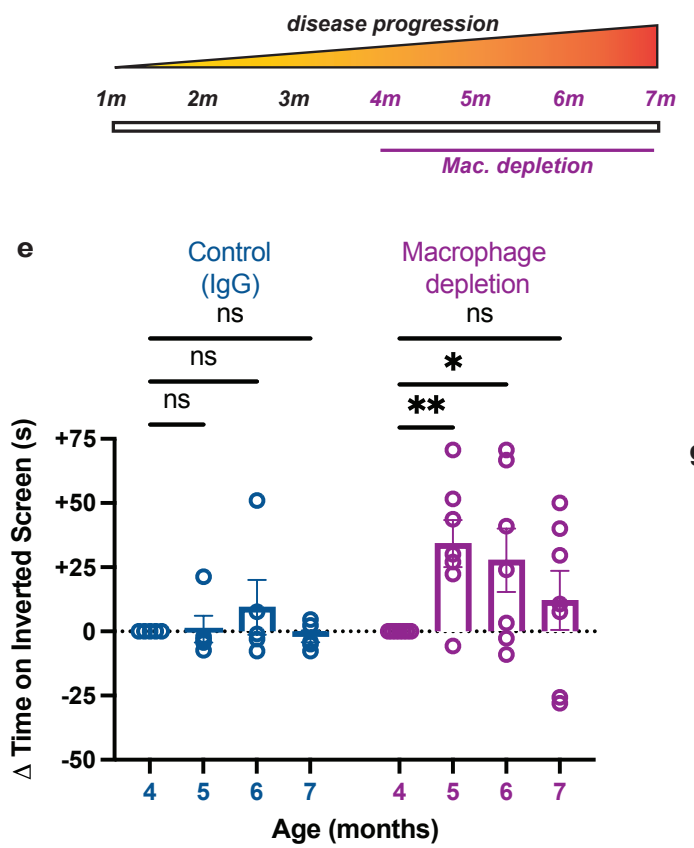

$\mathbf{f}$

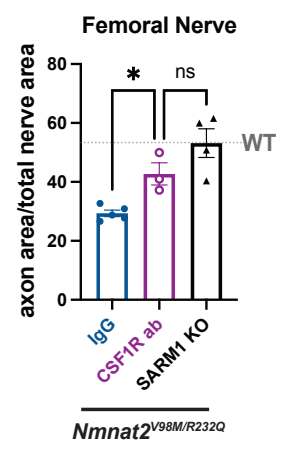

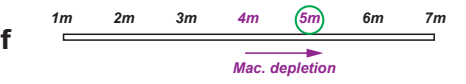

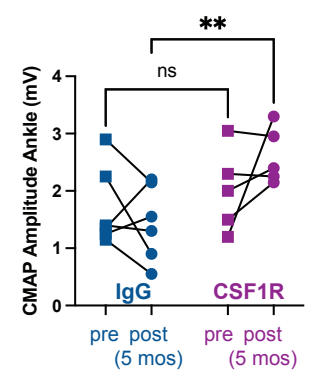

g
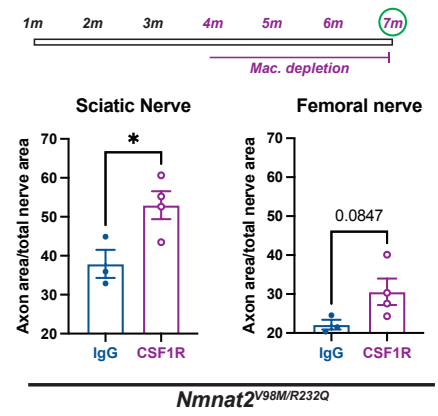

1226 Figure 6: Macrophage depletion rescues motor defects and axon loss in Nmnat2 ${ }^{\text {V98M/R232Q }}$ mice. a, Schematic of CSF1R antibody-mediated macrophage depletion in young (1 month) Nmnat2 ${ }^{V 98 M / R 232 Q}$ mice. $\mathbf{b}$, Average time suspended from an inverted screen (max. 120s) for IgG control and CSF1R-treated Nmnat2 ${ }^{V 98 M / R 232 Q}$ mice. Statistical significance was determined by two-way ANOVA with multiple comparisons. c, Representative images of femoral nerve from IgG (control), CSF1R, or Sarm1 KO $N$ mnat $2^{V 98 M / R 232 Q}$ mice at 4 months. Scale bar represents $150 \mu \mathrm{m}$. Percent axonal area as a ratio of total nerve area for femoral nerve calculated to the right. $\mathbf{d}$, Schematic of CSF1R antibody-mediated macrophage depletion in aged (4 months) $N$ mnat $2^{V 98 M / R 232 Q}$ mice. e, Change in inverted screen time (s) from pre-treatment (4 months) measured at 5, 6, and 7 months of age comparing CSF1R treatment or IgG (control). Statistical significance was determined by two-way ANOVA with multiple comparisons. f, CMAP amplitude of 
1238 Nmnat2 ${ }^{V 98 M / R 232 Q}$ mice at the ankle before and after 1 month of CSF1R treatment or IgG 1239 treatment. $\mathbf{g}$, Percent axonal area as a ratio of total nerve area for femoral nerve and 1240 sciatic nerve calculated at 7 months of age (3 months of macrophage depletion or 1241 treatment with isotype control $\operatorname{lgG}$ ). $\mathbf{f}-\mathbf{g}$, Statistical significance was determined by 1242 Student's unpaired t-test. All data are presented as mean +/- SEM. ns: not significant, $1243{ }^{*} p<0.05,{ }^{* *} p<0.01,{ }^{* * *} p<0.001,{ }^{* * * *} p<0.0001$. 


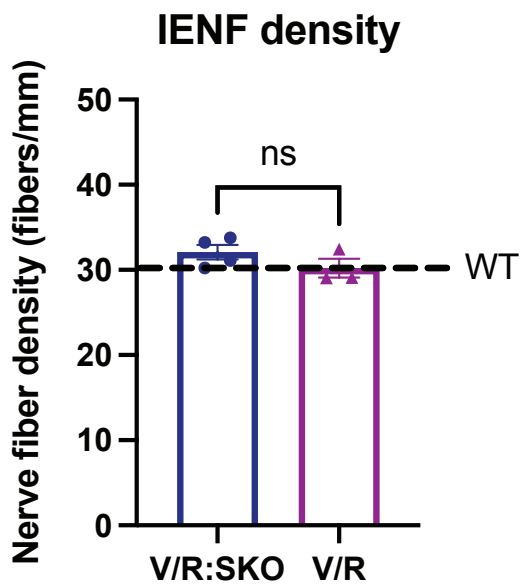
old Nmnat2 ${ }^{\mathrm{V} 98 M / R 232 Q}$; Sarm1 KO and Nmnat2 ${ }^{\mathrm{V} 98 M / R 232 Q}$ mice. 
1248
Sciatic Nerve

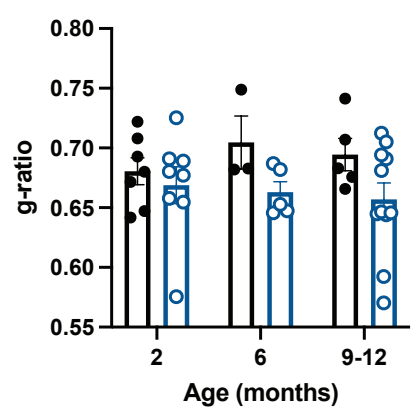

Femoral Nerve

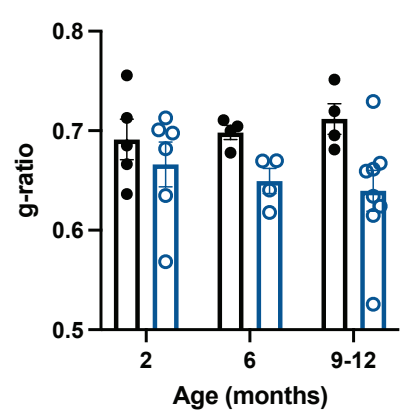

Sural Nerve

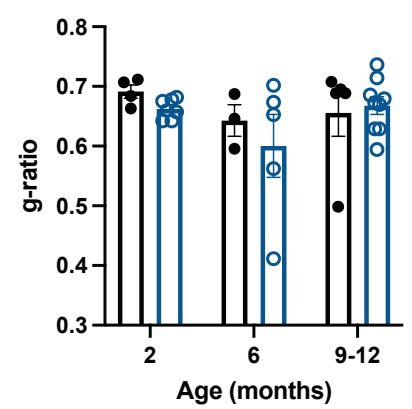

- WT

- $V 98 M / R 232 Q$

Supplemental Figure 2: Myelin thickness is preserved in Nmnat2 ${ }^{V 98 M / R 232 Q}$ mice. Average g-ratio in sciatic, femoral, and sural nerves at 2, 6, and 9-12 months of age, comparing Nmnat2 ${ }^{V 98 M / R 232 Q}$ mice to WT mice. All data are presented as mean +/- SEM. 
a
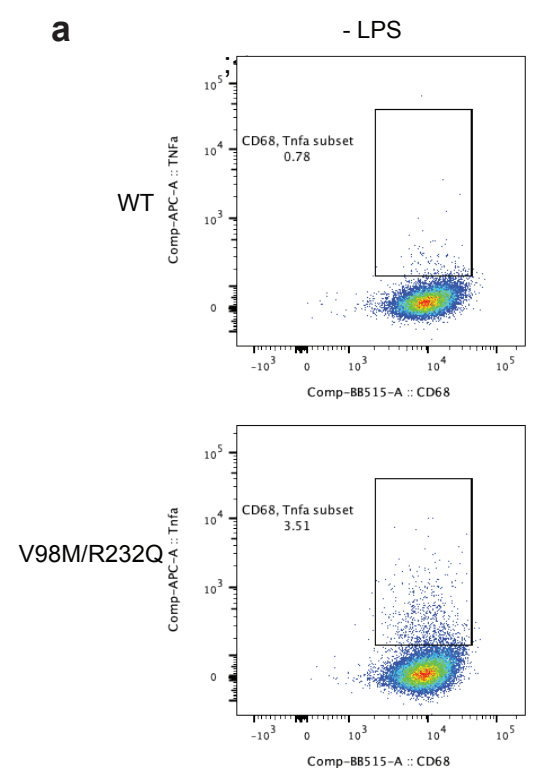

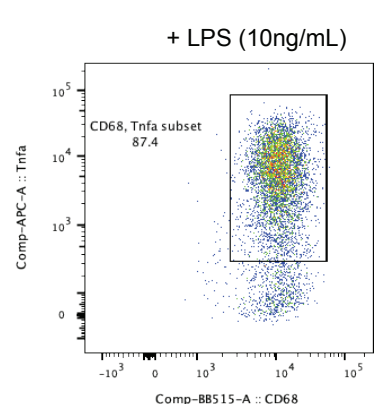

b
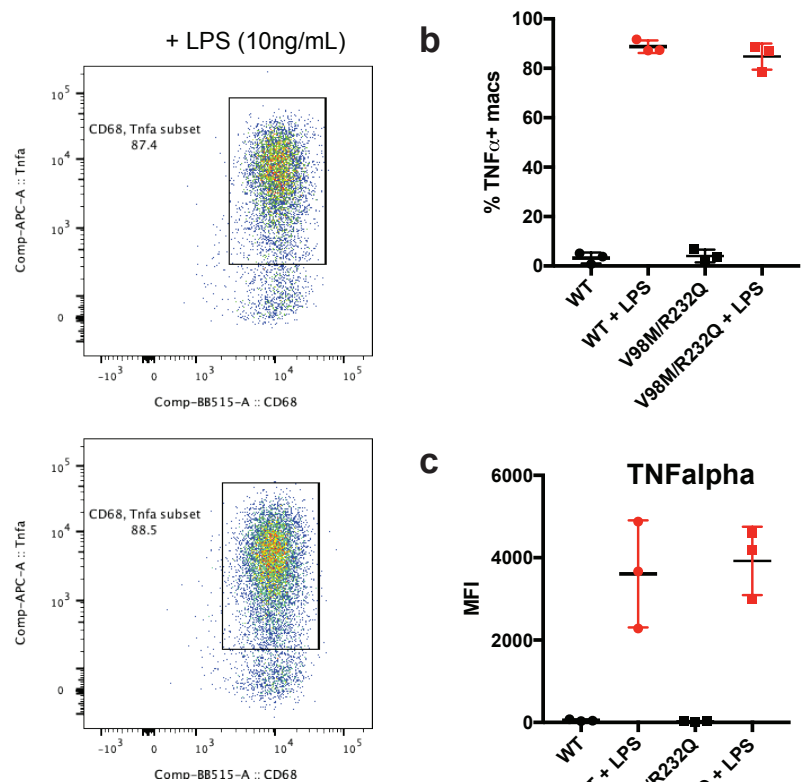

C

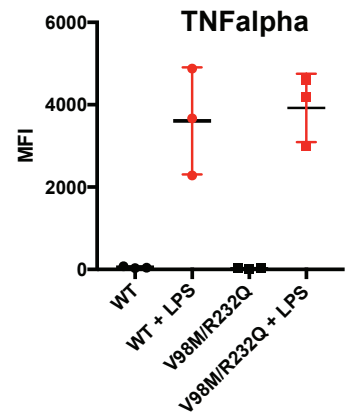

Supplemental Figure 3: Macrophage activation assay. a-b, Representative scatter plots (a) and quantification (b) of $\mathrm{CD} 68^{+} \mathrm{TNF}^{-a l p h a^{+}} \mathrm{Nmnat2}{ }^{\mathrm{V} 98 \mathrm{M} / \mathrm{R} 232 \mathrm{Q}}$ or WT peritoneal macrophages in the presence or absence of LPS $(10 \mathrm{ng} / \mathrm{mL})$ stimulation for $4 \mathrm{hrs}$. c, Mean fluorescence intensity (MFI) of TNF-alpha cell surface expression on CD68 ${ }^{+}$TNFalpha $^{+}$ peritoneal macrophages in the presence or absence of LPS $(10 \mathrm{ng} / \mathrm{mL})$ stimulation for 4 hrs. 

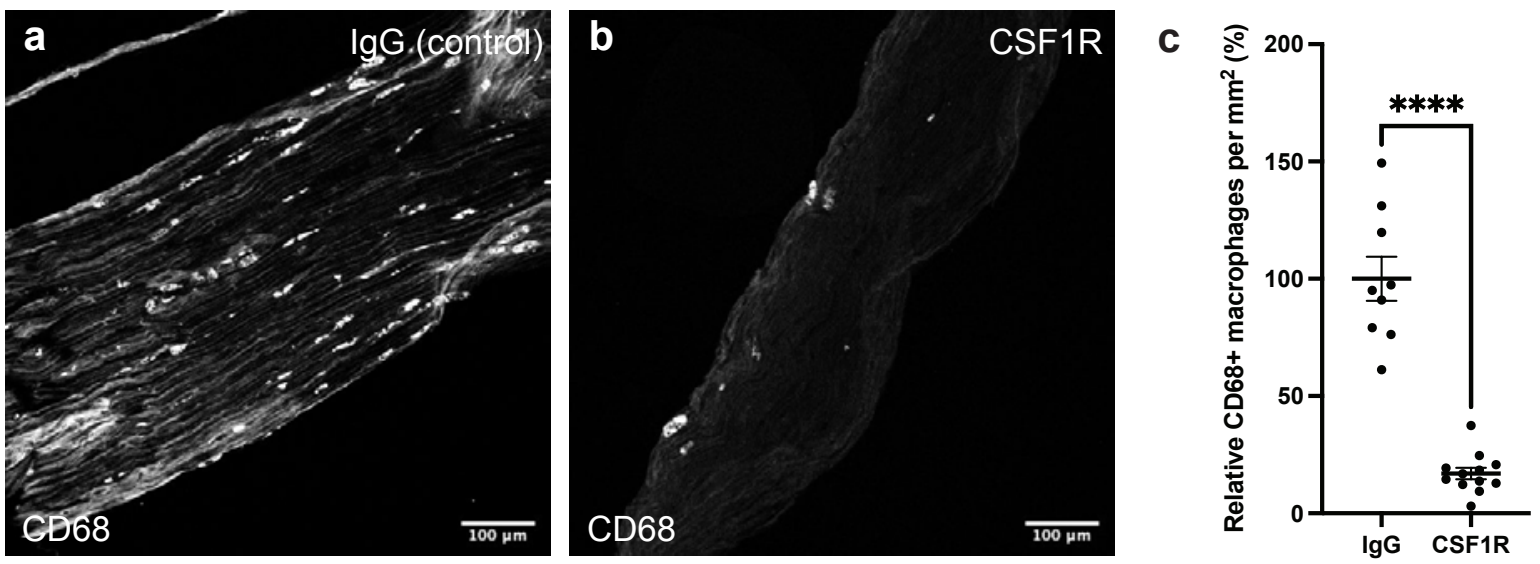

Supplemental Figure 4: Macrophage depletion quantification. a-b, Representative images of $\mathrm{CD}^{+} 8^{+}$cells in sciatic nerves after 3 months of CSF1R (a) or lgG (b) injections. c, Quantification of $\mathrm{CD}^{2} 8^{+}$cells per $\mathrm{mm}^{2}$ nerve area relative to $\mathrm{lgG}$ control nerves (reported as a percentage of control). 